\title{
A fast finite volume method for conservative space-fractional diffusion equations in convex domains
}

\author{
Jinhong Jia $^{a}$ Hong Wang ${ }^{b}$ \\ ${ }^{a}$ School of Mathematics, Shandong University, Jinan, Shandong 250100, China \\ ${ }^{b}$ Department of Mathematics, University of South Carolina, Columbia, South Carolina \\ 29208, USA (e-mail address: hwang@math.sc.edu, telephone: 803-777-4321. fax: \\ 803-777-6527)
}

\begin{abstract}
We develop a fast finite volume method for variable-coefficient, conservative space-fractional diffusion equations in convex domains via a volumepenalization approach. The method has an optimal storage and an almost linear computational complexity. The method retains second-order accuracy without requiring a Richardson extrapolation. Numerical results are presented to show the utility of the method.
\end{abstract}

Keywords: anomalous diffusion, circulant matrix, conjugate gradient squared method, fast Fourier transform, space-fractional diffusion equation, Toeplitz matrix, volume penalization

\section{Introduction}

Fractional partial differential equations (FPDEs) provide powerful alternatives to integer-order PDEs for modeling challenging phenomena such as anomalous transport, long-range interactions, and nonlocal dynamics [3, $8,21,23]$. However, FPDEs involve complex integral operators with singular kernels. Consequently, their numerical discretizations tend to generate dense stiffness matrices, for which traditionally used direct solvers $[16,17,19,24,27]$ require $O\left(N^{2}\right)$ memory and $O\left(N^{3}\right)$ computations for a problem of size $N$. The significantly increased computational complexity and memory requirement render realistic multidimensional FPDE modeling and simulations computationally intractable.

Extensive effort has been made to develop efficient and accurate numerical methods for multidimensional FPDEs. Meerschaert et al [19, 27] devel- 
oped alternating direction implicit (ADI) finite difference methods for spacefractional diffusion equations in rectangular domains. It was later proved $[28,30]$ that the stiffness matrices of the finite difference methods for these FPDEs on rectangular domains possess block-Toeplitz-Circulant-block like structures. Consequently, fast finite difference methods with $O(N \log N)$ computational complexity (per Krylov subspace iteration) and $O(N)$ memory requirement were developed accordingly. Numerical results show significant improvement over traditional solvers.

However, several issues remain: (i) These methods apply only to FPDEs on rectangular domains, as the development inherently utilizes the tensor product structure of the stiffness matrices that in turn require the tensor product structure of the domain. (ii) These methods apply only to nonconservative FPDEs, which are not equivalent to conservative FPDEs that arise in many applications [3, 8]. (iii) The shifted Grünwald approximation $[19,20]$ for retaining the stability of the finite difference methods reduces the spatial accuracy to first-order. A Richardson extrapolation has to be used to recover the second-order accuracy [27]. Although subsequent high-order finite difference methods were developed [15], it is not clear whether they retain the monotonicity $[19,20,27]$.

In this paper we develop a fast finite volume method for variable-coefficient conservative space-fractional FPDEs in convex domains via a volume-penalized approach. In section 2 we formulate a finite volume scheme for FPDEs in a rectangular domain. In sections 3 and 4 we prove that the dense stiffness matrix $A$ can be expressed as a superposition of block-tridiagonal Toeplitzblock like matrices and block-Toeplitz-Circulant-block like matrices, which can be stored in $O(N)$ memory. In section 5 we prove that the matrix-vector multiplication by $A$ can be evaluated in $O(N \log N)$ computations. In section 6 we develop a preconditioner for the numerical scheme. As it is not clear how the optimal-order superfast preconditioner presented in [29] for the finite volume method for one-dimensional FPDEs can be extended to the current two-dimensional problems, we present an efficient preconditioner based on the T Chan's circulant preonditioner and the proved structure of $A$. In section 7 we adopt a volume penalization approach, which was previously developed for modeling solid walls as porous media by letting permeability tends to zero $[13,14,22]$, to extend the fast finite volume method to FPDEs in general convex domains. In section 8 we carry out numerical experiments to investigate the performance of the fast finite volume method, which show the utility of the method. 


\section{A triangular finite volume scheme for FPDEs on a rectangular domain}

We consider the homogeneous Dirichlet boundary-value problem of the conservative space-fractional diffusion equation

$$
\begin{aligned}
& -D_{x}\left(K_{x}(x, y)\left(\gamma_{x} I_{x}^{\alpha}+\left(1-\gamma_{x}\right)_{x} I_{1}^{\alpha}\right) D_{x} u\right) \\
& -D_{y}\left(K_{y}(x, y)\left(\gamma_{y} I_{y}^{\beta}+\left(1-\gamma_{y}\right)_{y} I_{1}^{\beta}\right) D_{y} u\right)=f(x, y), \quad(x, y) \in \Omega, \\
& \quad u(x, y)=0, \quad(x, y) \in \partial \Omega .
\end{aligned}
$$

Here $\Omega$ is a rectangular domain in the plane that is assumed to be the unit square for the simplicity of notations, $D_{x}, K_{x}$, and $0 \leq \gamma_{x} \leq 1$ and $D_{y}, K_{y}$, and $0 \leq \gamma_{y} \leq 1$ represent the first-order differential operators, the diffusivity coefficients, and the relative weights of forward versus backward transition probability in the $x$ and $y$ directions, respectively, $f(x, y)$ is the source and sink term. The left and right fractional integral operators ${ }_{0} I_{x}^{\alpha},{ }_{x} I_{1}^{\alpha},{ }_{0} I_{y}^{\beta}$, and ${ }_{x} y I_{1}^{\beta}$ are defined by $[23,26]$

$$
\begin{aligned}
& { }_{0} I_{x}^{\alpha} u(x, y):=\frac{1}{\Gamma(\alpha)} \int_{0}^{x}(x-s)^{\alpha-1} u(s, y) d s, \\
& { }_{x} I_{1}^{\alpha} u(x, y):=\frac{1}{\Gamma(\alpha)} \int_{x}^{1}(s-x)^{\alpha-1} u(s, y) d s, \\
& { }_{0} I_{y}^{\beta} u(x, y):=\frac{1}{\Gamma(\beta)} \int_{0}^{y}(y-s)^{\beta-1} u(x, s) d s, \\
& { }_{y} I_{1}^{\beta} u(x, y):=\frac{1}{\Gamma(\beta)} \int_{y}^{1}(s-y)^{\beta-1} u(x, s) d s,
\end{aligned}
$$

with $\Gamma$ being the Gamma function.

Let $N_{x}$ and $N_{y}$ be postivie integers, and $h_{x}:=1 /\left(N_{x}+1\right)$ and $h_{y}:=$ $1 /\left(N_{y}+1\right)$ be the mesh sizes. We define $x_{i}:=i h_{x}$ and $y_{j}:=j h_{y}$ for $i=0,1, \cdots, N_{x}+1$ and $j=0,1, \cdots, N_{y}+1$ to form a rectangular partition of $\Omega$. We then subdivide each rectangular cell $\left[x_{i-1}, x_{i}\right] \times\left[y_{j-1}, y_{j}\right]$ into two triangular cells by connecting the diagonal with vertices $\left(x_{i-1}, y_{j}\right)$ and $\left(x_{i}, y_{j-1}\right)$. Let $\left\{\phi_{i, j}\right\}_{1 \leq i \leq N_{x}, 1 \leq j \leq N_{y}}$ be the set of piecewise-linear pyramid functions that assume value 1 at the node $\left(x_{i}, y_{j}\right)$ and 0 at all the other nodes. Let $x_{k-\frac{1}{2}}:=\left(x_{k-1}+x_{k}\right) / 2$ and $y_{l-\frac{1}{2}}:=\left(y_{l-1}+y_{l}\right) / 2$ for $k=1,2, \cdots, N_{x}+1$ and $l=1,2, \cdots, N_{y}+1$. We incorporate the numerical solution $u(x, y)$ of 
the form

$$
u(x, y)=\sum_{i=1}^{N_{x}} \sum_{j=1}^{N_{y}} u_{i, j} \phi_{i, j}(x, y)
$$

into equation (1), integrate the resulting equation on the control volume $\left[x_{k-\frac{1}{2}}, x_{k+\frac{1}{2}}\right] \times\left[y_{l-\frac{1}{2}}, y_{l+\frac{1}{2}}\right]$ and apply the divergence theorem, and approximate the integrand $K$ on each side by its value at the center of the side to obtain the following finite volume scheme

$$
\begin{gathered}
-\sum_{j=1}^{N_{y}} \sum_{i=1}^{N_{x}} u_{i, j}\left[K_{x}\left(x, y_{l}\right) \int_{y_{l-\frac{1}{2}}}^{y_{l+\frac{1}{2}}}\left(\gamma_{x}{ }_{0} I_{x}^{\alpha}+\left(1-\gamma_{x}\right){ }_{x} I_{1}^{\alpha}\right) D_{x} \phi_{i, j}(x, y) d y\right]||_{x_{k-\frac{1}{2}}}^{x_{k+\frac{1}{2}}} \\
-\left.\sum_{j=1}^{N_{y}} \sum_{i=1}^{N_{x}} u_{i, j}\left[K_{y}\left(x_{k}, y\right) \int_{x_{k-\frac{1}{2}}}^{x_{k+\frac{1}{2}}}\left(\gamma_{y}{ }_{0} I_{y}^{\beta}+\left(1-\gamma_{y}\right){ }_{y} I_{1}^{\beta}\right) D_{y} \phi_{i, j}(x, y) d x\right]\right|_{y_{l-\frac{1}{2}}} ^{y_{l+\frac{1}{2}}} \\
=\int_{x_{k-1 / 2}}^{x_{k+1 / 2}} \int_{y_{j-1 / 2}}^{y_{j+1 / 2}} f(x, y) d x d y, \quad 1 \leq k \leq N_{x}, \quad 1 \leq l \leq N_{y} .
\end{gathered}
$$

Let $N:=N_{x} N_{y}$. Let $u$ and $f$ be the $N$-dimensional vectors

$$
\begin{aligned}
u & =\left[u_{1,1}, \cdots, u_{N_{x}, 1}, u_{1,2}, \cdots, u_{N_{x}, 2}, u_{1, N_{y}}, \cdots, u_{N_{x}, N_{y}}\right]^{T}, \\
f & =\left[f_{1,1}, \cdots, f_{N_{x}, 1}, f_{1,2}, \cdots, f_{N_{x}, 2}, f_{1, N_{y}}, \cdots, f_{N_{x}, N_{y}}\right]^{T} .
\end{aligned}
$$

Let $A$ be the corresponding $N$-by- $N$ stiffness matrix. Then the finite volume scheme (3) can be expressed in the following matrix form

$$
A u=f
$$

The stiffness matrix $A$ is dense and has a complicated structure due to the nonlocal nature of the fractional integral operators. To develop an efficient and accurate finite volume method, we need to study the structure of $A$, by decomposing $A$ based on its coupling in the $x$ and $y$ directions, respectively

$$
A=A^{x}+A^{y}=\left(A_{l, j}^{x}\right)_{l, j=1}^{N_{y}}+\left(A_{l, j}^{y}\right)_{l, j=1}^{N_{y}}
$$

where all the matrix blocks $A_{l, j}^{x}$ and $A_{l, j}^{y}$ are matrices of order $N_{x}$ for $1 \leq$ 
$l, j \leq N_{y}$, and their entries are given by

$$
\begin{aligned}
\left(A_{l, j}^{x}\right)_{k, i} & =-\left.\left[K_{x}\left(x, y_{l}\right) \int_{y_{l-\frac{1}{2}}}^{y_{l+\frac{1}{2}}}\left(\gamma_{x}{ }_{0} I_{x}^{\alpha}+\left(1-\gamma_{x}\right){ }_{x} I_{1}^{\alpha}\right) D_{x} \phi_{i, j}(x, y) d y\right]\right|_{x_{k-\frac{1}{2}}} ^{x_{k+\frac{1}{2}}}, \\
\left(A_{l, j}^{y}\right)_{k, i} & =-\left.\left[K_{y}\left(x_{k}, y\right) \int_{x_{k-\frac{1}{2}}}^{x_{k+\frac{1}{2}}}\left(\gamma_{y}{ }_{0} I_{y}^{\beta}+\left(1-\gamma_{y}\right)_{y} I_{1}^{\beta}\right) D_{y} \phi_{i, j}(x, y) d x\right]\right|_{y_{l-\frac{1}{2}}} ^{y_{l+\frac{1}{2}}} .
\end{aligned}
$$

\section{Structure and efficient storage of matrix $A^{x}$}

Let $T^{(n)}\left(t_{n-1}, \ldots, t_{1} ; t_{0} ; t_{-1}, \ldots, t_{1-n}\right)$ denote an $n$-by-n Toeplitz matrix generated by $2 n-1$ parameters $\left\{t_{i}\right\}_{i=1-n}^{n-1}$

$$
\begin{aligned}
& T^{(n)}\left(t_{n-1}, \ldots, t_{1} ; t_{0} ; t_{-1}, \ldots, t_{1-n}\right) \\
& :=\left[\begin{array}{cccccc}
t_{0} & t_{-1} & t_{-2} & \ldots & t_{2-n} & t_{1-n} \\
t_{1} & t_{0} & t_{-1} & \ddots & \ddots & t_{2-n} \\
t_{2} & t_{1} & t_{0} & \ddots & \ddots & \vdots \\
\vdots & \ddots & \ddots & \ddots & t_{-1} & t_{-2} \\
t_{n-2} & \ddots & \ddots & t_{1} & t_{0} & t_{-1} \\
t_{n-1} & t_{n-2} & \ldots & t_{2} & t_{1} & t_{0}
\end{array}\right] .
\end{aligned}
$$

Here $t_{0}$ between two semicolons represents the diagonal entry. Let

$$
\begin{array}{ll}
K_{k, l}^{x,-}:=K_{x}\left(x_{k-\frac{1}{2}}, y_{l}\right), & K_{l}^{x,-}:=\left[K_{1, l}^{x,-}, K_{2, l}^{x,-}, \cdots, K_{N_{x}, l}^{x,-}\right]^{T} \\
K_{k, l}^{x,+}:=K_{x}\left(x_{k+\frac{1}{2}}, y_{l}\right), & K_{l}^{x,+}:=\left[K_{1, l}^{x,+}, K_{2, l}^{x,+}, \cdots, K_{N_{x}, l}^{x,+}\right]^{T} \\
& 1 \leq k \leq N_{x}, \quad 1 \leq l \leq N_{y} .
\end{array}
$$

Theorem 1. The matrix $A^{x}$ in (6) has a block tridiagonal structure, and each of its diagonal blocks can be decomposed as

$$
\begin{aligned}
A_{l, l}^{x}= & \frac{h_{y}}{\Gamma(\alpha+1) h_{x}^{1-\alpha}}\left[\operatorname{diag}\left(K_{l}^{x,-}\right)\left(\gamma_{x} D_{L,-}^{\left(\alpha, N_{x}\right)}+\left(1-\gamma_{x}\right) D_{R,-}^{\left(\alpha, N_{x}\right)}\right)\right. \\
& \left.-\operatorname{diag}\left(K_{l}^{x,+}\right)\left(\gamma_{x} D_{L,+}^{\left(\alpha, N_{x}\right)}+\left(1-\gamma_{x}\right) D_{R,+}^{\left(\alpha, N_{x}\right)}\right)\right], \quad 1 \leq l \leq N_{y} .
\end{aligned}
$$


Here $D_{L,-}^{\left(\alpha, N_{x}\right)}, D_{L,+}^{\left(\alpha, N_{x}\right)}, D_{R,-}^{\left(\alpha, N_{x}\right)}$, and $D_{R,+}^{\left(\alpha, N_{x}\right)}$ are Toeplitz matrices of the form

$$
\begin{aligned}
D_{L,-}^{\left(\alpha, N_{x}\right)} & :=T^{\left(N_{x}\right)}\left(c_{N_{x}-2}^{(\alpha)}, \cdots, c_{0}^{(\alpha)} ; c_{-1}^{\alpha} ; 0, \cdots, 0\right), \\
D_{L,+}^{\left(\alpha, N_{x}\right)} & :=T^{\left(N_{x}\right)}\left(c_{N_{x}-1}^{(\alpha)} \cdots, c_{1}^{(\alpha)} ; c_{0}^{(\alpha)} ; c_{-1}^{(\alpha)}, 0, \cdots, 0\right), \\
D_{R,-}^{\left(\alpha, N_{x}\right)} & :=T^{\left(N_{x}\right)}\left(0, \cdots, 0, d_{0}^{(\alpha)} ; d_{-1}^{(\alpha)} ; d_{-2}^{(\alpha)}, \cdots, d_{-N_{x}}^{(\alpha)}\right), \\
D_{R,+}^{\left(\alpha, N_{x}\right)} & :=T^{\left(N_{x}\right)}\left(0, \cdots, 0 ; d_{0}^{(\alpha)} ; d_{-1}^{(\alpha)}, \cdots, d_{1-N_{x}}^{(\alpha)}\right),
\end{aligned}
$$

where the entries $c_{N_{x}-1}^{(\alpha)}, c_{N_{x}-2}^{(\alpha)}, \cdots, c_{-1}^{(\alpha)}$ and $d_{0}^{(\alpha)}, d_{-1}^{(\alpha)}, \cdots, d_{-N_{x}}^{(\alpha)}$ are given by

$$
c_{m}^{(\alpha)}:= \begin{cases}\left(1+\frac{1}{\alpha+1}\right)\left(\frac{1}{2}\right)^{\alpha+1}, & m=-1, \\ \frac{1}{2}\left[\left(\frac{3}{2}\right)^{\alpha}-\left(\frac{1}{2}\right)^{\alpha-1}\right]+\frac{1}{\alpha+1}\left[\left(\frac{3}{2}\right)^{\alpha+1}-2\right], & m=0, \\ \frac{1}{2}\left[\left(m+\frac{3}{2}\right)^{\alpha}-2\left(m+\frac{1}{2}\right)^{\alpha}+\left(m-\frac{1}{2}\right)^{\alpha}\right] & \\ +\frac{1}{\alpha+1}\left[\left(m+\frac{3}{2}\right)^{\alpha+1}-\left(m-\frac{1}{2}\right)^{\alpha}\right. & \\ \left.+2 m^{\alpha+1}-2(m+1)^{\alpha+1}\right], & m \geq 1\end{cases}
$$

and

$$
d_{-m}^{(\alpha)}:= \begin{cases}\frac{1}{2}\left[2\left(m-\frac{1}{2}\right)^{\alpha}-\left(m+\frac{1}{2}\right)^{\alpha}-\left(m-\frac{3}{2}\right)^{\alpha}\right] & \\ +\frac{1}{\alpha+1}\left[\left(m-\frac{3}{2}\right)^{\alpha+1}-\left(m+\frac{1}{2}\right)^{\alpha+1}\right. & \\ \left.-2(m-1)^{\alpha+1}+2 m^{\alpha+1}\right], & m \geq 2, \\ -\frac{1}{2}\left[\left(\frac{3}{2}\right)^{\alpha}-\left(\frac{1}{2}\right)^{\alpha-1}\right]-\frac{1}{\alpha+1}\left[\left(\frac{3}{2}\right)^{\alpha+1}-2\right], & m=1, \\ -\left(1+\frac{1}{\alpha+1}\right)\left(\frac{1}{2}\right)^{\alpha+1}, & m=0 .\end{cases}
$$

The super-diagonal blocks of matrix $A^{x}$ can be decomposed as

$$
\begin{aligned}
A_{l, l+1}^{x} & :=\frac{h_{y}}{\Gamma(\alpha+1) h_{x}^{1-\alpha}}\left[\operatorname{diag}\left(K_{l}^{x,-}\right)\left(\gamma_{x} U_{L,-}^{\left(\alpha, N_{x}\right)}+\left(1-\gamma_{x}\right) U_{R,-}^{\left(\alpha, N_{x}\right)}\right)\right. \\
- & \left.\operatorname{diag}\left(K_{l}^{x,+}\right)\left(\gamma_{x} U_{L,+}^{\left(\alpha, N_{x}\right)}+\left(1-\gamma_{x}\right) U_{R,+}^{\left(\alpha, N_{x}\right)}\right)\right], \quad 1 \leq l \leq N_{y}-1
\end{aligned}
$$


Here $U_{L,-}^{\left(\alpha, N_{x}\right)}, U_{L,+}^{\left(\alpha, N_{x}\right)}, U_{R,-}^{\left(\alpha, N_{x}\right)}$, and $U_{R,+}^{\left(\alpha, N_{x}\right)}$ are Toeplitz matrices of the form

$$
\begin{aligned}
U_{L,-}^{\left(\alpha, N_{x}\right)} & :=T^{\left(N_{x}\right)}\left(a_{N_{x}-2}^{(\alpha)}, \cdots, a_{0}^{(\alpha)} ; 0 ; 0, \cdots, 0\right), \\
U_{L,+}^{\left(\alpha, N_{x}\right)} & :=T^{\left(N_{x}\right)}\left(a_{N_{x}-1}^{(\alpha)}, \cdots, a_{1}^{(\alpha)} ; a_{0}^{(\alpha)} ; 0, \cdots, 0\right), \\
U_{R,-}^{\left(\alpha, N_{x}\right)} & :=T^{\left(N_{x}\right)}\left(0, \cdots, 0, b_{0}^{(\alpha)} ; b_{-1}^{(\alpha)} ; b_{-2}^{(\alpha)}, \cdots, b_{-N_{x}}^{(\alpha)}\right), \\
U_{R,+}^{\left(\alpha, N_{x}\right)} & :=T^{\left(N_{x}\right)}\left(0, \cdots, 0 ; b_{0}^{(\alpha)} ; b_{-1}^{(\alpha)}, \cdots, b_{1-N_{x}}^{(\alpha)}\right),
\end{aligned}
$$

where the entries $a_{N_{x}-1}^{(\alpha)}, a_{N_{x}-2}^{(\alpha)}, \cdots, a_{0}^{(\alpha)}$ and $b_{0}^{(\alpha)}, b_{-1}^{(\alpha)}, \cdots, b_{-N_{x}}^{(\alpha)}$ are given by

$$
a_{m}^{(\alpha)}:= \begin{cases}-\left(1+\frac{1}{\alpha+1}\right)\left(\frac{1}{2}\right)^{\alpha+1}+\frac{1}{\alpha+1}, & m=0, \\ -\frac{1}{2}\left[\left(m+\frac{1}{2}\right)^{\alpha}-\left(m-\frac{1}{2}\right)^{\alpha}\right]+\frac{1}{\alpha+1}\left[(m+1)^{\alpha+1}\right. & \\ \left.-m^{\alpha+1}-\left(m+\frac{1}{2}\right)^{\alpha+1}+\left(m-\frac{1}{2}\right)^{\alpha+1}\right], & m \geq 1 .\end{cases}
$$

and

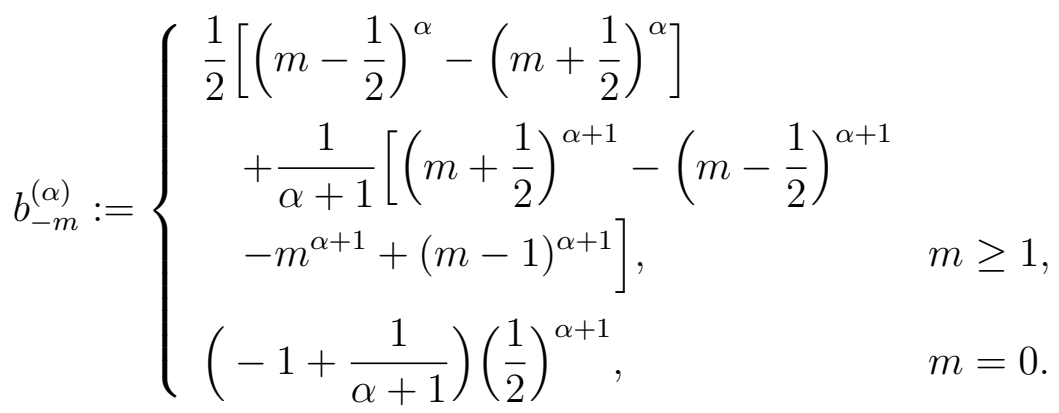

Finally, the sub-diagonal blocks of matrix $A^{x}$ can be decomposed as

$$
\begin{aligned}
A_{l, l-1}^{x}= & \frac{h_{y}}{\Gamma(\alpha+1) h_{x}^{1-\alpha}}\left[\operatorname{diag}\left(K_{l}^{x,-}\right)\left(\gamma_{x} L_{L,-}^{\left(\alpha, N_{x}\right)}+\left(1-\gamma_{x}\right) L_{R,-}^{\left(\alpha, N_{x}\right)}\right)\right. \\
& \left.-\operatorname{diag}\left(K_{l}^{x,+}\right)\left(\gamma_{x} L_{L,+}^{\left(\alpha, N_{x}\right)}+\left(1-\gamma_{x}\right) L_{R,+}^{\left(\alpha, N_{x}\right)}\right)\right], \quad 2 \leq l \leq N_{y} .
\end{aligned}
$$

Here $L_{L,-}^{\left(\alpha, N_{x}\right)}, L_{L,+}^{\left(\alpha, N_{x}\right)}, L_{R,-}^{\left(\alpha, N_{x}\right)}$, and $L_{R,+}^{\left(\alpha, N_{x}\right)}$ are Toeplitz matrices, and are related to $U_{L,-}^{\left(\alpha, N_{x}\right)}, U_{L,+}^{\left(\alpha, N_{x}\right)}, U_{R,-}^{\left(\alpha, N_{x}\right)}$, and $U_{R,+}^{\left(\alpha, N_{x}\right)}$ in (13) by

$$
\begin{array}{ll}
L_{L,-}^{\left(\alpha, N_{x}\right)}=-\left(U_{R,+}^{\left(\alpha, N_{x}\right)}\right)^{T}, & L_{L,+}^{\left(\alpha, N_{x}\right)}=-\left(U_{R,-}^{\left(\alpha, N_{x}\right)}\right)^{T}, \\
L_{R,-}^{\left(\alpha, N_{x}\right)}=-\left(U_{L,+}^{\left(\alpha, N_{x}\right)}\right)^{T}, & L_{R,+}^{\left(\alpha, N_{x}\right)}=-\left(U_{L,-}^{\left(\alpha, N_{x}\right)}\right)^{T} .
\end{array}
$$


Proof. We expand the first equation in (7) as

$$
\begin{aligned}
\left(A_{l, j}^{x}\right)_{k, i}= & \gamma_{x} K_{x}\left(x_{k-\frac{1}{2}}, y_{l}\right) \int_{y_{l-\frac{1}{2}}}^{y_{l+\frac{1}{2}}}{ }_{0} I_{x}^{\alpha} D_{x} \phi_{i, j}\left(x_{k-\frac{1}{2}}, y\right) d y \\
& -\gamma_{x} K_{x}\left(x_{k+\frac{1}{2}}, y_{l}\right) \int_{y_{l-\frac{1}{2}}}^{y_{l+\frac{1}{2}}}{ }_{0} I_{x}^{\alpha} D_{x} \phi_{i, j}\left(x_{k+\frac{1}{2}}, y\right) d y \\
& +\left(1-\gamma_{x}\right) K_{x}\left(x_{k+\frac{1}{2}}, y_{l}\right) \int_{y_{l-\frac{1}{2}}{ }_{x}{ }_{l+\frac{1}{2}}} I_{1}^{\alpha} D_{x} \phi_{i, j}\left(x_{k+\frac{1}{2}}, y\right) d y \\
& -\left(1-\gamma_{x}\right) K_{x}\left(x_{k-\frac{1}{2}}, y_{l}\right) \int_{y_{l-\frac{1}{2}}}^{y_{l+\frac{1}{2}}}{ }_{x} I_{1}^{\alpha} D_{x} \phi_{i, j}\left(x_{k-\frac{1}{2}}, y\right) d y \\
& 1 \leq k, i \leq N_{x}, \quad 1 \leq l, j \leq N_{y} .
\end{aligned}
$$

Hence, we need to evaluate the integrals of the left and right fractional derivatives on the intervals $\left[y_{l-\frac{1}{2}}, y_{l+\frac{1}{2}}\right]$ for $x=x_{k-\frac{1}{2}}$ and $x_{k+\frac{1}{2}}$, respectively.

Although $\phi_{i, j}$ is supported inside $\left[x_{i-1}, x_{i+1}\right] \times\left[y_{j-1}, y_{j+1}\right]$, its fractional derivative with respect to $x$ couples all the nodes in the $x$ direction. Hence,

$$
\left(\operatorname{supp}\left({ }_{0} I_{x}^{\alpha} D_{x} \phi_{i, j}\right) \bigcup \operatorname{supp}\left({ }_{x} I_{1}^{\alpha} D_{x} \phi_{i, j}\right)\right) \subset[0,1] \times\left[y_{j-1}, y_{j+1}\right] .
$$

We combine this with (16) to conclude that the matrix blocks satisfy

$$
A_{l, j}^{x}=0 \quad \forall|j-l| \geq 2, \quad 1 \leq l, j \leq N_{y} .
$$

That is, $A^{x}$ is a block-tridiagonal matrix.

The proof of (10), (12), and (14) is carried out via a direct verification, and is presented in the appendices (section 9.1).

Corollary 2. The matrix $A^{x}$ can be stored in $N+4 N_{x}+N_{y}+2$ (instead of $\left.N^{2}\right)$ memory.

Proof. By Theorem 1 and (9), we need to store $K_{x}\left(x_{k-\frac{1}{2}}, y_{l}\right)$ for $k=1,2, \ldots, N_{x}+$ 1 and $l=1,2, \ldots, N_{y}$ that requires $\left(N_{x}+1\right) N_{y}=N+N_{y}$ memory. In addition, we need to store $\left\{a_{m}^{(\alpha)}\right\}_{m=0}^{N_{x}-1},\left\{b_{-m}^{(\alpha)}\right\}_{m=0}^{N_{x}},\left\{c_{m}^{(\alpha)}\right\}_{m=-1}^{N_{x}-1}$, and $\left\{d_{-m}^{(\alpha)}\right\}_{m=0}^{N_{x}-1}$ that require $4 N_{x}+2$ memory. 


\section{Structure and efficient storage of matrix $A^{y}$}

We rewrite the second equation in (7) as follows

$$
\begin{aligned}
\left(A_{l, j}^{y}\right)_{k, i}= & -\left.\left[K_{y}\left(x_{k}, y\right) \int_{x_{k-\frac{1}{2}}}^{x_{k+\frac{1}{2}}}\left(\gamma_{y}{ }_{0} I_{y}^{\beta}+\left(1-\gamma_{y}\right){ }_{y} I_{1}^{\beta}\right) D_{y} \phi_{i, j}(x, y) d x\right]\right|_{y_{l-\frac{1}{2}}} ^{y_{l+\frac{1}{2}}} \\
= & \gamma_{y} K_{y}\left(x_{k}, y_{l-\frac{1}{2}}\right) \int_{x_{k-\frac{1}{2}}}^{x_{k}} I_{y}^{\beta} D_{y} \phi_{i, j}\left(x, y_{l-\frac{1}{2}}\right) d x \\
& -\gamma_{y} K_{y}\left(x_{k}, y_{l+\frac{1}{2}}\right) \int_{x_{k-\frac{1}{2}}}^{x_{k+\frac{1}{2}}}{ }_{0} I_{y}^{\beta} D_{y} \phi_{i, j}\left(x, y_{l+\frac{1}{2}}\right) d x \\
& +\left(1-\gamma_{y}\right) K_{y}\left(x_{k}, y_{l-\frac{1}{2}}\right) \int_{x_{k-\frac{1}{2}}{ }_{k+\frac{1}{2}}}^{{ }_{y}} I_{1}^{\beta} D_{y} \phi_{i, j}\left(x, y_{l-\frac{1}{2}}\right) d x \\
& -\left(1-\gamma_{y}\right) K_{y}\left(x_{k}, y_{l+\frac{1}{2}}\right) \int_{x_{k-\frac{1}{2}}}^{x_{k+\frac{1}{2}}}{ }_{y} I_{1}^{\beta} D_{y} \phi_{i, j}\left(x, y_{l+\frac{1}{2}}\right) d x .
\end{aligned}
$$

We let the $n$-by- $n$ matrices

$$
\begin{aligned}
& I^{(n)}:=T^{(n)}(0, \cdots, 0,0 ; 1 ; 0,0, \cdots, 0), \quad I_{-}^{(n)}:=T^{(n)}(0, \cdots, 0,1 ; 0 ; 0,0, \cdots, 0), \\
& I_{+}^{(n)}:=T^{(n)}(0, \cdots, 0,0 ; 0 ; 1,0, \cdots, 0)
\end{aligned}
$$

be the identity matrix, the matrix in which the 1's in $I^{(n)}$ are shifted to the subdiagonal, and the matrix in which the 1's in $I^{(n)}$ are shifted to the superdiagonal, respectively. Furthermore, we let

$$
\begin{array}{ll}
K_{k, l}^{y,-}:=K_{y}\left(x_{k}, y_{l-\frac{1}{2}}\right), & K_{k}^{y,-}:=\left[K_{k, 1}^{y,-}, K_{k, 2}^{y,-}, \cdots, K_{k, N_{y}}^{y,-}\right]^{T}, \\
K_{k, l}^{y,+}:=K_{y}\left(x_{k}, y_{l+\frac{1}{2}}\right), & K_{k}^{y,+}:=\left[K_{k, 1}^{y,+}, K_{k, 2}^{y,+}, \cdots, K_{k, N_{y}}^{y,+}\right]^{T} \\
& 1 \leq k \leq N_{x}, 1 \leq l \leq N_{y} .
\end{array}
$$

Although the role of $x$ and $y$ in equation (1) is symmetric, the index in the $x$ direction is labelled first. Consequently, the matrix $A^{y}$ has a different structure from $A^{x}$. We are now in the position to prove the following theorem.

Theorem 3. The matrix $A^{y}$ in (7) is a full block matrix, with each of its blocks being a tridiagonal matrix. Furthermore, matrix $A^{y}$ can be expressed 
as

$$
\begin{aligned}
A^{y}= & \frac{h_{x}}{\Gamma(\beta+1) h_{y}^{1-\beta}} \operatorname{diag}\left(K_{k}^{y,-}\right)\left[\left(\gamma_{y} L_{L,-}^{\left(\beta, N_{y}\right)}+\left(1-\gamma_{y}\right) L_{R,-}^{\left(\beta, N_{y}\right)}\right) \otimes I_{-}^{\left(N_{x}\right)}\right. \\
& +\left(\gamma_{y} D_{L,-}^{\left(\beta, N_{y}\right)}+\left(1-\gamma_{y}\right) D_{R,-}^{\left(\beta, N_{y}-1\right)}\right) \otimes I^{\left(N_{x}\right)} \\
& \left.+\left(\gamma_{y} U_{L,-}^{\left(\beta, N_{y}\right)}+\left(1-\gamma_{y}\right) U_{R,-}^{\left(\beta, N_{y}\right)}\right) \otimes I_{+}^{\left(N_{x}\right)}\right] \\
& -\frac{h_{x}}{\Gamma(\beta+1) h_{y}^{1-\beta}} \operatorname{diag}\left(K_{k}^{y,+}\right)\left[\left(\gamma_{y} L_{L,+}^{\left(\beta, N_{y}\right)}+\left(1-\gamma_{y}\right) L_{R,+}^{\left(\beta, N_{y}\right)}\right) \otimes I_{-}^{\left(N_{x}\right)}\right. \\
& +\left(\gamma_{y} D_{L,+}^{\left(\beta, N_{y}\right)}+\left(1-\gamma_{y}\right) D_{R,+}^{\left(\beta, N_{y}-1\right)}\right) \otimes I^{\left(N_{x}\right)} \\
& \left.+\left(\gamma_{y} U_{L,+}^{\left(\beta, N_{y}\right)}+\left(1-\gamma_{y}\right) U_{R,+}^{\left(\beta, N_{y}\right)}\right) \otimes I_{+}^{\left(N_{x}\right)}\right] .
\end{aligned}
$$

Here the matrices $L_{L,-}^{\left(\beta, N_{y}\right)}, L_{R,-}^{\left(\beta, N_{y}\right)}, L_{L,+}^{\left(\beta, N_{y}\right)}, L_{R,+}^{\left(\beta, N_{y}\right)}, D_{L,-}^{\left(\beta, N_{y}\right)}, D_{R,-}^{\left(\beta, N_{y}\right)}, D_{L,+}^{\left(\beta, N_{y}\right)}$, $D_{R,+}^{\left(\beta, N_{y}\right)}, U_{L,-}^{\left(\beta, N_{y}\right)}, U_{R,-}^{\left(\beta, N_{y}\right)}, U_{L,+}^{\left(\beta, N_{y}\right)}$, and $U_{R,+}^{\left(\beta, N_{y}\right)}$ are Toeplitz matrices defined in (11), (13), and (15) with $\alpha$ and $N_{x}$ being replaced by $\beta$ and $N_{y}$, respectively.

Proof. As $\operatorname{supp}\left\{\phi_{i, j}\right\} \subset\left[x_{i-1}, x_{i+1}\right] \times\left[y_{j-1}, y_{j+1}\right]$ for $i=1,2, \ldots, N_{x}$ and $j=1,2, \ldots, N_{y}$, its fractional derivative with respect to $y$ couples all the nodes in the $y$ direction. Hence,

$$
\left(\operatorname{supp}\left({ }_{0} I_{y}^{\beta} D_{y} \phi_{i, j}\right) \bigcup \operatorname{supp}\left({ }_{y} I_{1}^{\beta} D_{y} \phi_{i, j}\right)\right) \subset\left[x_{i-1}, x_{i+1}\right] \times[0,1] .
$$

We conclude from (17) and (20) that each matrix block $A_{l, j}^{y}$ in the matrix $A^{y}$ is nonzero. That is, the matrix $A^{y}$ is a full block matrix. Furthermore, we observe again from (17) and (20) that

$$
\left(A_{l, j}^{y}\right)_{k, i}=0, \quad|k-i| \geq 2, \quad l, j=1,2, \ldots, N_{y} .
$$

That is, each matrix block $A_{l, j}^{y}$ is a tridiagonal matrix for $l, j=1,2, \ldots, N_{y}$.

The proof for the rest of the theorem is presented in the appendices (section 9.2).

Corollary 4. The matrix $A^{y}$ can be stored in $N+N_{x}+4 N_{y}+2$ memory.

Proof. Although $A^{y}$ has a different decomposition from $A^{x}$, from Theorem 4 and (18) that we need to store $K_{y}\left(x_{k}, y_{l-\frac{1}{2}}\right)$ for $l=1,2, \ldots, N_{y}+1$ and $k=1,2, \ldots, K_{x}$ that requires $N_{x}\left(N_{y}+1\right)=N+N_{x}$ memory. In addition, we need to store $\left\{a_{m}^{(\beta)}\right\}_{m=0}^{N_{y}-1},\left\{b_{-m}^{(\beta)}\right\}_{m=0}^{N_{y}},\left\{c_{m}^{(\beta)}\right\}_{m=-1}^{N_{y}-1}$, and $\left\{d_{-m}^{(\beta)}\right\}_{m=0}^{N_{y}-1}$ that require $4 N_{y}+2$ memory. 


\section{A fast matrix-vector multiplication of $A u$}

Theorem 5. $A^{x} u$ can be evaluated in $O(N \log N)$ operations for any vector $u \in \mathbb{R}^{N}$.

Proof. Any $u \in \mathbb{R}^{N}$ can be expressed in the form of (4), which can be reformulated in the following block form

$$
u=\left[u_{1}^{T}, u_{2}^{T}, \cdots, u_{N_{y}}^{T}\right]^{T}, \quad u_{j}=\left[u_{1, j}, u_{2, j}, \cdots, u_{N_{x}, j}\right]^{T} .
$$

By Theorem 1, $A^{x}$ is a block tridiagonal matrix.

$$
A^{x} u=\left[\begin{array}{c}
A_{1,1}^{x} u_{1}+A_{1,2}^{x} u_{2} \\
A_{2,1}^{x} u_{1}+A_{2,2}^{x} u_{2}+A_{2,3}^{x} u_{3} \\
\vdots \\
A_{l, l-1}^{x} u_{l-1}+A_{l, l}^{x} u_{l}+A_{l, l+1}^{x} u_{l+1} \\
\vdots \\
A_{N_{y}-1, N_{y}-2}^{x} u_{N_{y}-2}+A_{N_{y}-1, N_{y}-1}^{x} u_{N_{y}-1}+A_{N_{y}-1, N_{y}}^{x} u_{N_{y}} \\
A_{N_{y}, N_{y}-1}^{x} u_{N_{y}-1}+A_{N_{y}, N_{y}}^{x} u_{N_{y}}
\end{array}\right] .
$$

To prove the theorem, we count the computational complexity of $A_{l, j}^{x} u_{j}$ for $j=l-1, l$, and $l+1$. Without loss of generality, we consider the computational complexity of $A_{l, l}^{x} u_{l}$. By (10), the matrix-vector multiplication by diagonal matrix of order $N_{x}$ is $O\left(N_{x}\right)$. We need only to focus on the matrix-vector multiplication by $D_{L,-}^{\left(\alpha, N_{x}\right)}, D_{R,-}^{\left(\alpha, N_{x}\right)}, D_{L,+}^{\left(\alpha, N_{x}\right)}$, and $D_{R,+}^{\left(\alpha, N_{x}\right)}$, which are Toeplitz matrices by Theorem 1 .

It is known that a Toeplitz matrix $T^{(n)}$ (of the form (8)) can be embedded into a $2 n$-by- $2 n$ circulant matrix $[7,12]$

$$
C_{2 n}=\left[\begin{array}{ll}
T^{(n)} & S^{(n)} \\
S^{(n)} & T^{(n)}
\end{array}\right], \quad S^{(n)}:=T^{(n)}\left(t_{-1}, \ldots, t_{1-n} ; 0 ; t_{n-1}, \ldots, t_{1}\right),
$$

and $C^{(2 n)}$ can be diagonalized by the Fourier transform matrix $F^{(2 n)}$

$$
C^{(2 n)}=\left(F^{(2 n)}\right)^{-1} \operatorname{diag}\left(F^{(2 n)} c^{(2 n)}\right) F^{(2 n)}
$$

where $c^{(2 n)}$ is the first column vector of $C^{(2 n)}$. The matrix-vector multiplication $F^{(2 n)} w^{(2 n)}$ for any vector $w^{(2 n)} \in \mathbb{R}^{2 n}$ can be carried out in $O(2 n \log (2 n))=$ $O(n \log (n))$ operations by the fast discrete Fourier transform (FFT). Thus, 
$C^{(2 n)} w^{(2 n)}$ can be evaluated in $O(n \log (n))$ operations. For any vector $w^{(n)} \in$ $\mathbb{R}^{n}$, by choosing $w^{(2 n)}:=\left[w_{n}^{T}, 0^{T}\right]^{T}, T^{(n)} w^{(n)}$ can be evaluated in $O(n \log n)$ operations.

In the current context, $D_{L,-}^{\left(\alpha, N_{x}\right)} u_{l}, D_{R,-}^{\left(\alpha, N_{x}\right)} u_{l}, D_{L,+}^{\left(\alpha, N_{x}\right)} u_{l}$, and $D_{R,+}^{\left(\alpha, N_{x}\right)} u_{l}$ can be evaluated in $O\left(N_{x} \log N_{x}\right)$ operations. By (10), $A_{l, l}^{x} u_{l}$ can be evaluated in $O\left(N_{x} \log N_{x}\right)$ operations. Similarly, by (12) and (14), we conclude that $A_{l, l-1}^{x} u_{l-1}$ and $A_{l, l+1}^{x} u_{l+1}$ can also be evaluated in $O\left(N_{x} \log N_{x}\right)$ operations. We conclude from (22) that $A^{x} u$ can be evaluated in $N_{y} O\left(N_{x} \log N_{x}\right)=$ $O(N \log N)$ operations.

Theorem 6. $A^{y} u$ can be evaluated in $O(N \log N)$ operations for any $u \in \mathbb{R}^{N}$.

Proof. By (19) in Theorem 2, the matrix $A^{y}$ can be decomposed as a finite sum of diagonal matrices multiplied by a block-Toeplitz-circulant-block matrix. Without loss of generality, we take a representative term $L_{L,-}^{\left(\beta, N_{y}\right)} \otimes I_{-}^{\left(N_{x}\right)}$ in (19) to show the procedure.

We first embed $L_{L,-}^{\left(\beta, N_{y}\right)} \otimes I_{-}^{\left(N_{x}\right)}$ into a $2 N$-by- $2 N$ block-circulant-circulantblock matrix and a $2 N$-dimensional vector $u^{(2 N)} \in \mathbb{R}^{2 N}$

$$
C_{B}^{(2 N)}:=\left[\begin{array}{cc}
L_{L,-}^{\left(\beta, N_{y}\right)} \otimes I_{-}^{\left(N_{x}\right)} & S_{L,-}^{\left(\beta, N_{y}\right)} \otimes I_{-}^{\left(N_{x}\right)} \\
S_{L,-}^{\left(\beta, N_{y}\right)} \otimes I_{-}^{\left(N_{x}\right)} & L_{L,-}^{\left(\beta, N_{y}\right)} \otimes I_{-}^{\left(N_{x}\right)}
\end{array}\right], \quad u^{(2 N)}:=\left[\begin{array}{l}
u \\
0
\end{array}\right],
$$

where $S_{L,-}^{\left(\beta, N_{y}\right)}$ is a Toeplitz matrix as defined in (23), in which $T^{(n)}$ is replaced by $L_{L,-}^{\left(\beta, N_{y}\right)}$.

Let $c_{B}^{(2 N)}$ be its first column vector. The corresponding two-dimensional discrete Fourier transform matrix is $F^{\left(2 N_{y}\right)} \otimes F^{\left(N_{x}\right)}$. The Fourier transform $\hat{c}_{B}^{(2 N)}$ of $c_{B}^{(2 N)}$ is given by

$$
\hat{c}_{B}^{(2 N)}:=\left(F^{\left(2 N_{y}\right)} \otimes F^{\left(N_{x}\right)}\right) c_{B}^{(2 N)} .
$$

Any block-circulant-circulant-block matrix $C_{B}^{(2 N)}$ can be diagonalized by the Fourier transform matrix [7, 12]

$$
C_{B}^{(2 N)}=\left(F^{\left(2 N_{y}\right)} \otimes F^{\left(N_{x}\right)}\right)^{-1} \operatorname{diag}\left(\hat{c}_{B}^{(2 N)}\right)\left(F^{\left(2 N_{y}\right)} \otimes F^{\left(N_{x}\right)}\right) .
$$

It is well known that for any vector $u^{(2 N)} \in \mathbb{R}^{2 N}$ the matrix-vector multiplication $\left(F^{\left(2 N_{y}\right)} \otimes F^{\left(N_{x}\right)}\right) u^{(2 N)}$ can be evaluated in $O(2 N \log (2 N))=O(N \log N)$ 
operations via the fast discrete Fourier transform (FFT). (26) shows that $C_{B}^{(2 N)} u^{(2 N)}$ can be evaluated in $O(N \log N)$ operations. The first $N$ equations in $C_{B}^{(2 N)} u^{(2 N)}$ yields $\left(L_{L,-}^{\left(\beta, N_{y}\right)} \otimes I_{-}^{\left(N_{x}\right)}\right) u$. By $(19), A^{y} u$ can be evaluated in $O(N \log N)$ operations.

Corollary 7. Au can be evaluated in $O(N \log N)$ operations for any vector $u \in \mathbb{R}^{N}$.

Proof. It is a direct conclusion of Theorems 5 and 6 as well as (6).

\section{An efficient and robust preconditioner}

The application of the fast matrix-vector multiplication algorithm presented in section 5 to any Krylov subspace iterative solver directly leads to a fast version of the solver. In this section we further develop a preconditioned version that helps further reducing the number of iterations.

The development is based on the T. Chan's circulant preconditioner for Toeplitz systems $[5,6]$. Theorem 5 shows that the matrix $A^{x}$ can be decomposed as a finite sum of diagonal matrices multiplied by block-tridiagonal, block-Toeplitz-Toeplitz-block matrices. On the other hand, Theorem 6 shows that the matrix $A^{y}$ can be decomposed as a finite sum of diagonal matrices multiplied by block-Toeplitz-circulant-block matrices. Although in any fast Krylov subspace method $A^{x} u$ and $A^{y} u$ can be evaluated separately in $O(N \log N)$ operations by Theorems 5 and 6 , a preconditioner for $A^{x}+A^{y}$ has to be developed as a whole.

To do so, we let $\bar{K}_{x}$ and $\bar{K}_{y}$ be constant approximations (e.g., the mean) of $K_{x}(x, y)$ and $K_{y}(x, y)$, and let $\bar{A}^{x}$ and $\bar{A}^{y}$ be defined by the expressions (10), (12), (14), and (19) with $K_{x}(x, y)$ and $K_{y}(x, y)$ being replaced by $\bar{K}_{x}$ and $\bar{K}_{y}$, respectively. Then

$$
\bar{A}:=\bar{A}_{x}+\bar{A}_{y}
$$

is a block-Toeplitz-Toeplitz-block matrix of the form

$$
\bar{A}=\left[\begin{array}{lllll}
T_{0}^{\left(N_{x}\right)} & T_{-1}^{\left(N_{x}\right)} & \ldots & T_{2-N_{y}}^{\left(N_{x}\right)} & T_{1-N_{y}}^{\left(N_{x}\right)} \\
T_{1}^{\left(N_{x}\right)} & T_{0}^{\left(N_{x}\right)} & \ldots & T_{3-N_{y}}^{\left(N_{x}\right)} & T_{2-N_{y}}^{\left(N_{x}\right)} \\
\vdots & \ddots & \ddots & \ddots & \vdots \\
T_{N_{y}-2}^{\left(N_{x}\right)} & \ddots & T_{1}^{\left(N_{x}\right)} & T_{0}^{\left(N_{x}\right)} & T_{-1}^{\left(N_{x}\right)} \\
T_{N_{y}-1}^{\left(N_{x}\right)} & T_{N_{y}-2}^{\left(N_{x}\right)} & \cdots & T_{1}^{\left(N_{x}\right)} & T_{0}^{\left(N_{x}\right)}
\end{array}\right]
$$


where each matrix block $T_{l}^{\left(N_{x}\right)}$ is a Toeplitz matrix of the form (8) with $n$ being replaced by $N_{x}$ and $t_{j}$ by $t_{l, j}$ for $j=1-N_{x}, \ldots,-1,0,1, \ldots, N_{x}-1$ and $l=1-N_{y}, \ldots,-1,0,1, \ldots, N_{y}-1$.

We apply the T. Chan's circulant preconditioner $C_{l}^{\left(N_{x}\right)}:=\left(c_{l, i-k}\right)_{1 \leq i, k<N_{x}}$ of $T_{l}^{\left(N_{x}\right)}[5,6]$ :

$$
c_{l, j}:=\left\{\begin{array}{cl}
\frac{\left(N_{x}-j\right) t_{l, j}+j t_{l, j-N_{x}}}{N_{x}}, & 0 \leq j \leq N_{x}-1, \\
c_{l, N_{x}+j}, & 1-N_{x} \leq j<0 .
\end{array}\right.
$$

We then define a circulant-block-block-circulant matrix $C_{B}^{(N)}:=\left(C_{q-p}\right)_{1 \leq p, q<N_{y}}$ to be the T. Chan's block-circulant preconditioner

$$
C_{k}:= \begin{cases}C_{0}^{\left(N_{x}\right)}, & k=0, \\ \frac{\left(N_{y}+k\right) C_{k}^{\left(N_{x}\right)}-k C_{N_{y}+k},}{N_{y}}, & 1-N_{y} \leq k<0, \\ C_{k-N_{y}}, & 0<k<N_{y} .\end{cases}
$$

The matrix $C_{B}^{(N)}$ is an $N_{y}$-by- $N_{y}$ block-circulant matrix with $N_{x}$-by- $N_{x}$ circulant blocks. We deduce from the construction of $C_{B}^{(N)}$ that its storage only requires $O(N)$ memory. Furthermore, we observe from $(26)$ that $C_{B}^{(N)}$ can be inverted in $O(N \log N)$ operations.

The combination of the preconditioner can be used with any fast Krylov subspace iterative solvers.

\section{A volume-penalized finite volume scheme for FPDEs on convex domains}

We consider an extension of problem (1) to a convex domain $\Omega_{s}$

$$
\begin{aligned}
& -D_{x}\left(K_{x}(x, y)\left(\gamma_{x} a_{1}(y) I_{x}^{\alpha}+\left(1-\gamma_{x}\right)_{x} I_{b_{1}(y)}^{\alpha}\right) D_{x} u\right) \\
& -D_{y}\left(K_{y}(x, y)\left(\gamma_{y} a_{2}(x) I_{y}^{\beta}+\left(1-\gamma_{y}\right)_{y} I_{b_{2}(x)}^{\beta}\right) D_{y} u\right)=f(x, y), \quad(x, y) \in \Omega_{s}, \\
& u(x, y)=0, \quad(x, y) \in \partial \Omega_{s} .
\end{aligned}
$$

A major difference of (27) from (1) is that the lower limits of the left fractional integrals and the upper ones of the right fractional integrals in $x$ and $y$ depend on $y$ or $x$, respectively. Here $a_{1}(y)$ and $b_{1}(y)$ represent the left and 
right boundary of $\Omega_{s}$ at given $y$, and $a_{2}(x)$ and $b_{2}(x)$ represent the lower and upper boundary of $\Omega_{s}$ at given $x$, which are used as the lower (right) limits of the left (right) fractional integral operators in (2).

Assume that problem (27) can be extended to a rectangular domain $\Omega=(a, b) \times(c, d)$ that contains $\Omega_{s}$. Then we formulate a volume-penalized homogeneous Dirichlet boundary-value problem on $\Omega$

$$
\begin{gathered}
-D_{x}\left(K_{x}(x, y)\left(\gamma_{x} a_{1}(y) I_{x}^{\alpha}+\left(1-\gamma_{x}\right){ }_{x} I_{b_{1}(y)}^{\alpha}\right) D_{x} u_{\eta}\right) \\
-D_{y}\left(K_{y}(x, y)\left(\gamma_{y} a_{2}(x) I_{y}^{\beta}+\left(1-\gamma_{y}\right){ }_{y} I_{b_{2}(x)}^{\beta}\right) D_{y} u_{\eta}\right) \\
+\frac{1-1_{\Omega_{s}}(x, y)}{\eta} u_{\eta}=f(x, y), \quad(x, y) \in \Omega, \\
u(x, y)=0, \quad(x, y) \in \partial \Omega .
\end{gathered}
$$

Here $1_{\Omega_{s}}$ is the indicator function of the domain $\Omega_{s}$, which equals to 1 if $(x, y) \in \Omega_{s}$ or 0 elsewhere. Hence, the FPDE in (28) reduces to that in (27) if $(x, y) \in \Omega_{s}$. However, the volume-penalized solution $u_{\eta}$ does not satisfy the homogeneous Dirichlet boundary condition on the boundary $\partial \Omega_{s}$. Heuristically,

$$
\lim _{\eta \rightarrow 0^{+}} u_{\eta}(x, y)=0, \quad(x, y) \in \Omega \backslash \Omega_{s} .
$$

That is, $u_{\eta}$ satisfies the FPDE in (27) and is expected to satisfy the homogernous volumetric Dirichlet boundary condition on $\Omega \backslash \Omega_{s}$ asymptotically. As a matter of fact, it was shown in $[1,4,11,13]$ that for many problems the solution of the penalized PDEs converges towards the solution of the original PDEs, while the error depends on the penalization parameter. We take these results as a proof of concept to develop a volume-penalized finite volume method for space-fractional diffusion equations in convex domains.

As problem (28) is a homogeneous Dirichlet boundary-value problem on a rectangular domain, the fast finite volume scheme in the previous sections applies. However, the last term on the left-hand side of (28) introduces an additional diagonal matrix to the stiffness matrix of the finite volume scheme to problem (1). The diagonal entries of this diagonal matrix can vary anywhere from 0 to a diagonal entry of the corresponding mass matrix multiplied by $1 / \eta$. Hence, this matrix is not Toeplitz, in general. Consequently, the preconditioner developed in section 6 does not apply. Additional work in the future needs to be conducted to develop an efficient preconditioner. 
Table 1: Performance of the Gauss, CGS, FCGS, and PFCGS solvers for the finite volume scheme for the example in $\S 8.1$.

\begin{tabular}{|c|c|c|c|c|}
\hline & $N_{x}=N_{y}$ & $\left\|u_{h}-u\right\|_{L^{2}}$ & \# of iter. & CPUs \\
\hline \multirow{3}{*}{ Gauss } & $2^{5}$ & $2.705367 \mathrm{E}-3$ & & $46 \mathrm{~s}$ \\
& $2^{6}$ & $6.793973 \mathrm{E}-4$ & & $1 \mathrm{~h} 2 \mathrm{~m}$ \\
& $2^{7}$ & $1.694831 \mathrm{E}-4$ & & $7 \mathrm{~d} 17 \mathrm{~h}$ \\
& $2^{8}$ & & out of memory & \\
\hline conv. rate & & $M=2.76, \kappa=2.00$ & & \\
\hline & $2^{5}$ & $2.705367 \mathrm{E}-3$ & 25 & $6.04 \mathrm{~s}$ \\
& $2^{6}$ & $6.793973 \mathrm{E}-4$ & 40 & $3 \mathrm{~m} 5 \mathrm{~s}$ \\
& $2^{7}$ & $1.694831 \mathrm{E}-4$ & 70 & $2 \mathrm{~h} 34 \mathrm{~m}$ \\
& $2^{8}$ & & out of memory & \\
\hline conv. rate & & $\mathrm{M}=2.76, \kappa=2.00$ & & \\
\hline & $2^{5}$ & $2.705367 \mathrm{E}-3$ & 24 & $0.48 \mathrm{~s}$ \\
& $2^{6}$ & $6.793973 \mathrm{E}-4$ & 37 & $1.53 \mathrm{~s}$ \\
& $2^{7}$ & $1.694831 \mathrm{E}-4$ & 60 & $12 \mathrm{~s}$ \\
& $2^{8}$ & $4.216027 \mathrm{E}-5$ & 92 & $49 \mathrm{~s}$ \\
& $2^{9}$ & & divergent & \\
\hline conv. rate & & $\mathrm{M}=2.79, \kappa=2.00$ & & \\
\hline & $2^{5}$ & $2.705367 \mathrm{E}-3$ & 11 & $0.28 \mathrm{~s}$ \\
PFCGS & $2^{6}$ & $6.793973 \mathrm{E}-4$ & 12 & $0.57 \mathrm{~s}$ \\
& $2^{7}$ & $1.694831 \mathrm{E}-4$ & 13 & $2.95 \mathrm{~s}$ \\
& $2^{8}$ & $4.216027 \mathrm{E}-5$ & 16 & $9.74 \mathrm{~s}$ \\
& $2^{9}$ & $1.047953 \mathrm{E}-5$ & 18 & $54 \mathrm{~s}$ \\
& $2^{10}$ & $2.605420 \mathrm{E}-6$ & 21 & $4 \mathrm{~m} 37 \mathrm{~s}$ \\
& $2^{11}$ & $6.481977 \mathrm{E}-7$ & 25 & $27 \mathrm{~m} 32 \mathrm{~s}$ \\
& $2^{12}$ & $1.610818 \mathrm{E}-7$ & 33 & $1 \mathrm{~h} 38 \mathrm{~m}$ \\
\hline conv. rate & & $\mathrm{M}=2.85, \kappa=2.01$ & & \\
\hline
\end{tabular}




\section{Numerical experiments}

We carry out numerical experiments to investigate the performance of the fast finite volume method for the homogeneous Dirichlet boundary-value problem of space-fractional FPDEs.

\subsection{Numerical simulation of FPDEs on a rectangular domain}

We simulate problem (1) by the finite-volume scheme (3), which is solved by Gaussian elimination (Gauss), the conjugate gradient squared (CGS) method, the fast CGS (FCGS) method, and the preconditioned fast CGS (PFCGS) method. The primary goal of this example is to investigate the computational efficiency and memory requirement of all these solvers.

In the numerical experiments we choose $\gamma_{x}=\gamma_{y}=0.5, \alpha=\beta=0.8$, and $K_{x}=K_{y}=1$. The solution is chosen to be $u(x, y):=256 x^{2}(1-x)^{2} y^{2}(1-y)^{2}$. The right-hand side is calculated accordingly

$$
\begin{aligned}
f(x, y)= & -256 y^{2}(1-y)^{2}\left[\frac{1}{\Gamma(\alpha+1)}\left(x^{\alpha}+(1-x)^{\alpha}\right)\right. \\
& \left.-\frac{6}{\Gamma(\alpha+2)}\left(x^{\alpha+1}+(1-x)^{\alpha+1}\right)+\frac{12}{\Gamma(\alpha+3)}\left(x^{\alpha+2}+(1-x)^{\alpha+2}\right)\right] \\
& -256 x^{2}(1-x)^{2}\left[\frac{1}{\Gamma(\beta+1)}\left(y^{\beta}+(1-y)^{\beta}\right)\right. \\
& \left.-\frac{6}{\Gamma(\beta+2)}\left(y^{\beta+1}+(1-y)^{\beta+1}\right)+\frac{12}{\Gamma(\beta+3)}\left(y^{\beta+2}+(1-y)^{\beta+2}\right)\right] .
\end{aligned}
$$

$\{\mathrm{Ne}\}$

We present the $L^{2}$ errors of the numerical solutions, the associated convergence rates

$$
\left\|u_{h}-u\right\|_{L^{2}} \leq M h^{\kappa},
$$

the number of iterations of the iteratives solvers, and the CPU time consumed by all the solvers in Table 1 .

We observe that these solvers generate identical numerical solutions with second-order convergence rate without using a Richardson extrapolation. Their major differences reside in the memory requirement that limits the size of a problem that can be simulated on a given machine and the CPU times they take. The largest size that can be solved by Gauss is $N_{x}=N_{y}=2^{7}$, i.e., about 32,000 triangular elements, which takes more than a week of CPU time to finish. This is also the largest problem size of the CGS can solve due to its limitation on the memory requirement. The FCGS solves the same problem using only 12 seconds of CPU time. Furthermore, the FCGS solves 
Table 2: Performance of the volume-penalized finite volume scheme for the example in $\S 8.2$ with $\alpha=\beta=0.1$

\begin{tabular}{|c|c|c|c|c|c|}
\hline & $N_{x}=N_{y}$ & $\mid u_{h}-u \|_{L^{2}\left(\Omega_{s}\right)}$ & $\left\|u_{h}\right\|_{L^{2}\left(\Omega \backslash \Omega_{s}\right)}$ & \# of iter. & CPUs \\
\hline \multirow{6}{*}{$\eta=20$} & $2^{4}$ & $5.105588 \mathrm{E}-3$ & $4.611015 \mathrm{E}-4$ & 27 & $0.23 \mathrm{~s}$ \\
\hline & $2^{5}$ & $1.165746 \mathrm{E}-3$ & $2.779546 \mathrm{E}-4$ & 50 & $0.79 \mathrm{~s}$ \\
\hline & $2^{6}$ & $3.326281 \mathrm{E}-4$ & $9.079025 \mathrm{E}-5$ & 101 & $3.24 \mathrm{~s}$ \\
\hline & $2^{7}$ & $9.642932 \mathrm{E}-5$ & $1.405125 \mathrm{E}-5$ & 160 & $16 \mathrm{~s}$ \\
\hline & $2^{8}$ & $2.508560 \mathrm{E}-5$ & $7.478186 \mathrm{E}-6$ & 307 & $1 \mathrm{~m} 12 \mathrm{~s}$ \\
\hline & $2^{9}$ & $6.907164 \mathrm{E}-6$ & $2.467309 \mathrm{E}-6$ & 592 & $14 \mathrm{~m} 17 \mathrm{~s}$ \\
\hline conv. rate & & $M=50, \kappa=1.88$ & $M=50, \kappa=1.60$ & & \\
\hline \multirow{6}{*}{$\eta=1$} & $2^{4}$ & $5.106374 \mathrm{E}-3$ & $4.589053 \mathrm{E}-4$ & 31 & $0.25 \mathrm{~s}$ \\
\hline & $2^{5}$ & $1.166439 \mathrm{E}-3$ & $2.731328 \mathrm{E}-4$ & 50 & $0.79 \mathrm{~s}$ \\
\hline & $2^{6}$ & $3.317735 \mathrm{E}-4$ & $8.890816 \mathrm{E}-5$ & 100 & $3.31 \mathrm{~s}$ \\
\hline & $2^{7}$ & $9.640066 \mathrm{E}-5$ & $1.396566 \mathrm{E}-5$ & 185 & $18 \mathrm{~s}$ \\
\hline & $2^{8}$ & $2.500944 \mathrm{E}-5$ & 7.381211E-6 & 308 & $1 \mathrm{~m} 12 \mathrm{~s}$ \\
\hline & $2^{9}$ & $6.865761 \mathrm{E}-6$ & $2.408850 \mathrm{E}-6$ & 591 & $16 \mathrm{~m} \mathrm{8s}$ \\
\hline conv. rate & & $M=50, \kappa=1.89$ & $M=50, \kappa=1.60$ & & \\
\hline \multirow{6}{*}{$\eta=0.1$} & $2^{4}$ & $5.169564 \mathrm{E}-3$ & $3.297783 \mathrm{E}-4$ & 29 & $0.38 \mathrm{~s}$ \\
\hline & $2^{5}$ & $1.209662 \mathrm{E}-3$ & $1.567743 \mathrm{E}-4$ & 56 & $1.51 \mathrm{~s}$ \\
\hline & $2^{6}$ & $3.178022 \mathrm{E}-4$ & $4.382610 \mathrm{E}-5$ & 93 & $5.99 \mathrm{~s}$ \\
\hline & $2^{7}$ & $9.424197 \mathrm{E}-5$ & $9.725284 \mathrm{E}-6$ & 169 & $20 \mathrm{~s}$ \\
\hline & $2^{8}$ & $2.327820 \mathrm{E}-5$ & $4.231561 \mathrm{E}-6$ & 325 & $2 \mathrm{~m} 7 \mathrm{~s}$ \\
\hline & $2^{9}$ & $6.149905 \mathrm{E}-6$ & $1.130637 \mathrm{E}-6$ & 625 & $22 \mathrm{~m} 12 \mathrm{~s}$ \\
\hline conv. rate & & $M=50, \kappa=1.93$ & $M=50, \kappa=1.68$ & & \\
\hline \multirow{6}{*}{$\eta=0.01$} & $2^{4}$ & $5.317458 \mathrm{E}-3$ & $1.289428 \mathrm{E}-5$ & 225 & $2.23 \mathrm{~s}$ \\
\hline & $2^{5}$ & $1.299251 \mathrm{E}-3$ & $1.360030 \mathrm{E}-5$ & 191 & $3.89 \mathrm{~s}$ \\
\hline & $2^{6}$ & $3.271512 \mathrm{E}-4$ & $5.886561 \mathrm{E}-6$ & 189 & $7.94 \mathrm{~s}$ \\
\hline & $2^{7}$ & $9.106917 \mathrm{E}-5$ & $2.067034 \mathrm{E}-6$ & 191 & $25 \mathrm{~s}$ \\
\hline & $2^{8}$ & $2.310850 \mathrm{E}-5$ & $8.687672 \mathrm{E}-7$ & 350 & $1 \mathrm{~m} \mathrm{57s}$ \\
\hline & $2^{9}$ & 6.124672E-6 & $2.414062 \mathrm{E}-7$ & 621 & $20 \mathrm{~m} \mathrm{25s}$ \\
\hline conv. rate & & $M=50, \kappa=1.95$ & $M=50, \kappa=1.20$ & & \\
\hline
\end{tabular}


the problem with 131,000 triangular elements using only 49 seconds of CPU time. However, the FCGS diverges with 524,000 triangular elements due to the greatly increased number of iterations that accumulates round-off errors. Finally, the PFCGS solves the problem with 32,000 triangular elements using only 2.95 seconds of CPU time. More importantly, the PFCGS still retains second-order accuracy of the finite volume scheme even with a very fine mesh of 33,554,000 triangular elements using only 1 hour 38 minutes of CPU time.

\subsection{Numerical simulation of FPDEs on a convex domain}

The purpose of this example is to investigate the convergence behavior of the volume-penalized finite volume method for different orders of fractional diffusions and different penalization parameters. Note that the numerical experiments in section 8.1 show that all the solvers generate almost identical numerical solutions but with significantly different computational efficiency and memory requirement. Hence, in this subsection we simulate problem (27) by the volume-penalized finite volume scheme solved by the FCGS solver.

In the numercal example runs, the domain $\Omega_{s}$ is chosen to be the unit disk centered at the origin, the diffusivity coefficients $K_{x}(x, y)=K_{y}(x, y)=0.005$, and $\gamma_{x}=\gamma_{y}=0.5$. The true solution $u$ is chosen to be $u(x, y)=\left(1-x^{2}-y^{2}\right)^{2}$ on $\Omega_{s}=\left\{(x, y): 1-x^{2}-y^{2}>0\right\}$. The physical domain $\Omega_{s}$ is extended to a square domain $\Omega=(-1,1) \times(-1,1)$. The orders $\alpha=\beta$ are chosen to be 0.1 , $0.5,0.8$, or 0.9 , respectively. The penalization parameter $\eta=20,1,0.1$, and 0.01 , respectively. The right-hand side term $f$ is presented in the appendices (section $\S 9.3$ ). We present the $L^{2}$ errors of the numerical solutions in the original physical domain $\Omega_{s}$ and the associated convergence rates, the $L^{2}$ norms of the numerical solutions in the extended domain $\Omega \backslash \Omega_{s}$, the number of iterations of the FCGS solver, and the CPU time consumed by the FCGS solver for $\eta=20,1,0.1$ and 0.01 in Tables 2-5, respectively, for $\alpha=\beta=0.1$, $0.5,0.8$, or 0.9 .

We have the following observations from these results: (i) The numerical solution of the penalized finite volume scheme (28) converges to the true solution of the fractional diffusion equation (27) in the original physical domain $\Omega_{s}$. (ii) The volume-penalized finite volume has approximately a second order convergence rate. (iii) The errors of the numerical solutions and the corresponding convergence rates are improved slightly for smaller $\eta$. (iv) The penalization errors, i.e., the $L^{2}$ norms of the numerical solutions on the extended domain $\Omega \backslash \Omega_{s}$, converges to zero, and are much smaller for a smaller $\eta$. However, the FCGS solver diverges for $\eta=0.001$ and $N_{x}=N_{y}=2^{6}$. 


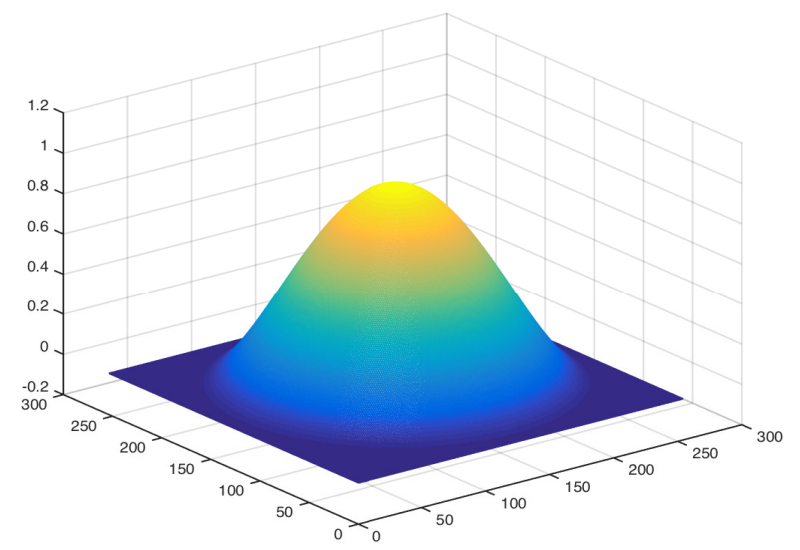

Figure 1: The numerical solution of $\S 8.2$ with $N=2^{8}, \alpha=\beta=0.1$ and $\eta=0.01$.

(v) Figure 1 shows that the volume-penalized solution retains the monotonic behavior of the trial solution. Figure 2 shows that the numerical errors occur primarily near the physical boundary of the convex domain $\Omega_{s}$. (vi) The FCGS solver for the volume-penalized finite volume scheme in general convex domains has a significantly larger number of iterations than that for the finite volume scheme in rectangular domains. Hence, the development of an effective and efficient preconditioner for the volume-penalized finite volume scheme is of great importance. Nevertheless, this turns out to be a highly nontrivial task and will be investigated in the future.

\section{Concluding remarks}

Conservative fractional diffusion partial differential equations appear in physical and other applications, in which local conservation property is crucial in almost all applications. We develop a fast finite volume scheme for a conservative space-fractional diffusion equation in a two-dimensional rectangular domain with a triangular partition, by exploring Toeplitz-like structures of the stiffness matrix of the numerical scheme. The scheme is locally conservative, and naturally has second-order accuracy without resorting to a Richardson extrapolation, as in the case of finite difference methods for fractional partial differential equations. The fast finite volume scheme reduces the storage from $O\left(N^{2}\right)$ to optimal order and computational cost from 
Table 3: Performance of the volume-penalized finite volume scheme for the example in $\S 8.2$ with $\alpha=\beta=0.5$

\begin{tabular}{|c|c|c|c|c|c|}
\hline & $N_{x}=N_{y}$ & $\mid u_{h}-u \|_{L^{2}\left(\Omega_{s}\right)}$ & $\left\|u_{h}\right\|_{L^{2}\left(\Omega \backslash \Omega_{s}\right)}$ & $\#$ of iter. & CPUs \\
\hline \multirow{6}{*}{$\eta=20$} & $2^{4}$ & $8.038725 \mathrm{E}-3$ & $1.012507 \mathrm{E}-3$ & 18 & $0.19 \mathrm{~s}$ \\
\hline & $2^{5}$ & $2.194657 \mathrm{E}-3$ & $2.454815 \mathrm{E}-4$ & 30 & $0.48 \mathrm{~s}$ \\
\hline & $2^{6}$ & $5.896355 \mathrm{E}-4$ & $5.756537 \mathrm{E}-5$ & 53 & $1.81 \mathrm{~s}$ \\
\hline & $2^{7}$ & $1.546495 \mathrm{E}-4$ & $9.607366 \mathrm{E}-6$ & 77 & $8.31 \mathrm{~s}$ \\
\hline & $2^{8}$ & 4.030678E-5 & $2.523522 \mathrm{E}-6$ & 129 & $33 \mathrm{~s}$ \\
\hline & $2^{9}$ & $1.032376 \mathrm{E}-5$ & $4.238125 \mathrm{E}-7$ & 218 & $4 \mathrm{~m} \mathrm{51s}$ \\
\hline conv. rate & & $M=50, \kappa=1.92$ & $M=50, \kappa=2.24$ & & \\
\hline \multirow{6}{*}{$\eta=1$} & $2^{4}$ & $8.041256 \mathrm{E}-3$ & $9.815132 \mathrm{E}-4$ & 21 & $0.20 \mathrm{~s}$ \\
\hline & $2^{5}$ & $2.194119 \mathrm{E}-3$ & $2.400594 \mathrm{E}-4$ & 30 & $0.51 \mathrm{~s}$ \\
\hline & $2^{6}$ & $5.892573 \mathrm{E}-4$ & $5.600322 \mathrm{E}-5$ & 52 & $1.73 \mathrm{~s}$ \\
\hline & $2^{7}$ & $1.545884 \mathrm{E}-4$ & $9.504617 \mathrm{E}-6$ & 77 & $7.84 \mathrm{~s}$ \\
\hline & $2^{8}$ & $4.028365 \mathrm{E}-5$ & $2.473514 \mathrm{E}-6$ & 128 & $30 \mathrm{~s}$ \\
\hline & $2^{9}$ & $1.032008 \mathrm{E}-5$ & 4.189462E-7 & 215 & $5 \mathrm{~m} \mathrm{21s}$ \\
\hline conv. rate & & $M=50, \kappa=1.92$ & $M=50, \kappa=2.24$ & & \\
\hline \multirow{6}{*}{$\eta=0.1$} & $2^{4}$ & $8.125784 \mathrm{E}-3$ & $3.048303 \mathrm{E}-4$ & 33 & $0.35 \mathrm{~s}$ \\
\hline & $2^{5}$ & $2.192787 \mathrm{E}-3$ & $1.195115 \mathrm{E}-4$ & 33 & $0.59 \mathrm{~s}$ \\
\hline & $2^{6}$ & $5.841575 \mathrm{E}-4$ & $3.107359 \mathrm{E}-5$ & 51 & $1.75 \mathrm{~s}$ \\
\hline & $2^{7}$ & $1.533719 \mathrm{E}-5$ & 6.993403E-6 & 80 & $8.42 \mathrm{~s}$ \\
\hline & $2^{8}$ & $3.985794 \mathrm{E}-5$ & $1.713294 \mathrm{E}-6$ & 133 & $31 \mathrm{~s}$ \\
\hline & $2^{9}$ & $1.024384 \mathrm{E}-5$ & $3.283857 \mathrm{E}-7$ & 202 & $4 \mathrm{~m} 17 \mathrm{~s}$ \\
\hline conv. rate & & $M=50, \kappa=2.02$ & $M=50, \kappa=1.99$ & & \\
\hline \multirow{6}{*}{$\eta=0.01$} & $2^{4}$ & $8.168134 \mathrm{E}-3$ & $4.749137 \mathrm{E}-6$ & 225 & $1.62 \mathrm{~s}$ \\
\hline & $2^{5}$ & $2.200145 \mathrm{E}-3$ & $3.526516 \mathrm{E}-6$ & 225 & $3.83 \mathrm{~s}$ \\
\hline & $2^{6}$ & $5.827862 \mathrm{E}-4$ & 1.679299E-6 & 277 & $9.06 \mathrm{~s}$ \\
\hline & $2^{7}$ & $1.522980 \mathrm{E}-4$ & $7.730844 \mathrm{E}-7$ & 306 & $31 \mathrm{~s}$ \\
\hline & $2^{8}$ & $3.933708 \mathrm{E}-5$ & $3.321876 \mathrm{E}-7$ & 259 & $59 \mathrm{~s}$ \\
\hline & $2^{9}$ & $1.009454 \mathrm{E}-5$ & 1.054429E-7 & 269 & $5 \mathrm{~m} \mathrm{49s}$ \\
\hline conv. rate & & $M=50, \kappa=1.93$ & $M=50, \kappa=1.11$ & & \\
\hline
\end{tabular}


Table 4: Performance of the volume-penalized finite volume scheme for the example in $\S 8.2$ with $\alpha=\beta=0.8$

\begin{tabular}{|c|c|c|c|c|c|}
\hline & $N_{x}=N_{y}$ & $\mid u_{h}-u \|_{L^{2}\left(\Omega_{s}\right)}$ & $\mid u_{h} \|_{L^{2}\left(\Omega \backslash \Omega_{s}\right)}$ & $\#$ of iter. & CPUs \\
\hline \multirow{6}{*}{$\eta=20$} & $2^{4}$ & $9.110784 \mathrm{E}-3$ & $1.293500 \mathrm{E}-3$ & 13 & $0.13 \mathrm{~s}$ \\
\hline & $2^{5}$ & $2.464426 \mathrm{E}-3$ & $2.778429 \mathrm{E}-4$ & 18 & $0.38 \mathrm{~s}$ \\
\hline & $2^{6}$ & $6.490006 \mathrm{E}-4$ & $5.692362 \mathrm{E}-5$ & 27 & $1.01 \mathrm{~s}$ \\
\hline & $2^{7}$ & $1.671521 \mathrm{E}-4$ & $1.078059 \mathrm{E}-5$ & 41 & $4.25 \mathrm{~s}$ \\
\hline & $2^{8}$ & $4.274620 \mathrm{E}-5$ & $2.241586 \mathrm{E}-6$ & 63 & $14 \mathrm{~s}$ \\
\hline & $2^{9}$ & $1.081652 \mathrm{E}-5$ & $3.872405 \mathrm{E}-7$ & 97 & $2 \mathrm{~m} \mathrm{1s}$ \\
\hline conv. rate & & $M=50, \kappa=1.95$ & $M=50, \kappa=2.34$ & & \\
\hline \multirow{6}{*}{$\eta=1$} & $2^{4}$ & $9.102824 \mathrm{E}-3$ & $1.128693 \mathrm{E}-3$ & 13 & $0.14 \mathrm{~s}$ \\
\hline & $2^{5}$ & $2.459318 \mathrm{E}-3$ & $2.561752 \mathrm{E}-4$ & 19 & $0.33 \mathrm{~s}$ \\
\hline & $2^{6}$ & $6.475784 \mathrm{E}-4$ & $5.371356 \mathrm{E}-5$ & 27 & $0.92 \mathrm{~s}$ \\
\hline & $2^{7}$ & 1.669042E-4 & $1.043037 \mathrm{E}-5$ & 40 & $4 \mathrm{~s}$ \\
\hline & $2^{8}$ & $4.268967 \mathrm{E}-5$ & $2.180501 \mathrm{E}-6$ & 61 & $14 \mathrm{~s}$ \\
\hline & $2^{9}$ & $1.080844 \mathrm{E}-5$ & $3.808157 \mathrm{E}-7$ & 94 & $1 \mathrm{~m} 58 \mathrm{~s}$ \\
\hline conv. rate & & $M=50, \kappa=1.95$ & $M=50, \kappa=2.30$ & & \\
\hline \multirow{6}{*}{$\eta=0.1$} & $2^{4}$ & $9.092794 \mathrm{E}-3$ & $1.020488 \mathrm{E}-4$ & 43 & $0.41 \mathrm{~s}$ \\
\hline & $2^{5}$ & $2.432835 \mathrm{E}-3$ & $4.877727 \mathrm{E}-5$ & 57 & $1.09 \mathrm{~s}$ \\
\hline & $2^{6}$ & $6.374089 \mathrm{E}-4$ & $1.575694 \mathrm{E}-5$ & 58 & $2.19 \mathrm{~s}$ \\
\hline & $2^{7}$ & 1.643669E-4 & $4.620875 \mathrm{E}-6$ & 55 & $6.66 \mathrm{~s}$ \\
\hline & $2^{8}$ & $4.205859 \mathrm{E}-5$ & $1.243207 \mathrm{E}-6$ & 65 & $17 \mathrm{~s}$ \\
\hline & $2^{9}$ & $1.069783 \mathrm{E}-5$ & $2.650807 \mathrm{E}-7$ & 93 & $2 \mathrm{~m} 14 \mathrm{~s}$ \\
\hline conv. rate & & $M=50, \kappa=1.95$ & $M=50, \kappa=1.73$ & & \\
\hline \multirow{6}{*}{$\eta=0.01$} & $2^{4}$ & $9.093917 \mathrm{E}-3$ & $1.125734 \mathrm{E}-6$ & 225 & $1.58 \mathrm{~s}$ \\
\hline & $2^{5}$ & $2.429707 \mathrm{E}-3$ & $6.640245 \mathrm{E}-7$ & 961 & $15 \mathrm{~s}$ \\
\hline & $2^{6}$ & $6.352265 \mathrm{E}-4$ & $2.791970 \mathrm{E}-7$ & 310 & $11 \mathrm{~s}$ \\
\hline & $2^{7}$ & $1.633605 \mathrm{E}-4$ & $1.194645 \mathrm{E}-7$ & 370 & $38 s$ \\
\hline & $2^{8}$ & $4.166470 \mathrm{E}-5$ & $5.271340 \mathrm{E}-8$ & 404 & $1 \mathrm{~m} 36 \mathrm{~s}$ \\
\hline & $2^{9}$ & $1.058670 \mathrm{E}-5$ & $1.944513 \mathrm{E}-8$ & 445 & $9 \mathrm{~m} \mathrm{35s}$ \\
\hline conv. rate & & $M=50, \kappa=1.95$ & $M=50, \kappa=1.73$ & & \\
\hline
\end{tabular}


Table 5: Performance of the volume-penalized finite volume scheme for the example in $\S 8.2$ with $\alpha=\beta=0.9$

\begin{tabular}{|c|c|c|c|c|c|}
\hline & $N_{x}=N_{y}$ & $\mid u_{h}-u \|_{L^{2}\left(\Omega_{s}\right)}$ & $\left\|u_{h}\right\|_{L^{2}\left(\Omega \backslash \Omega_{s}\right)}$ & \# of iter. & CPUs \\
\hline \multirow{6}{*}{$\eta=20$} & $2^{4}$ & $9.362172 \mathrm{E}-3$ & $1.362098 \mathrm{E}-3$ & 11 & $0.12 \mathrm{~s}$ \\
\hline & $2^{5}$ & $2.519421 \mathrm{E}-3$ & $2.851351 \mathrm{E}-4$ & 16 & $0.28 \mathrm{~s}$ \\
\hline & $2^{6}$ & $6.595694 \mathrm{E}-4$ & $5.736933 \mathrm{E}-5$ & 22 & $1.09 \mathrm{~s}$ \\
\hline & $2^{7}$ & $1.692336 \mathrm{E}-4$ & $1.094364 \mathrm{E}-5$ & 33 & $3.28 \mathrm{~s}$ \\
\hline & $2^{8}$ & 4.309593E-5 & $2.227257 \mathrm{E}-6$ & 49 & $11 \mathrm{~s}$ \\
\hline & $2^{9}$ & $1.088034 \mathrm{E}-5$ & $3.846701 \mathrm{E}-7$ & 71 & $1 \mathrm{~m} \mathrm{30s}$ \\
\hline conv. rate & & $M=50, \kappa=1.95$ & $M=50, \kappa=2.35$ & & \\
\hline \multirow{6}{*}{$\eta=1$} & $2^{4}$ & $9.340856 \mathrm{E}-3$ & $1.006460 \mathrm{E}-3$ & 12 & $0.13 \mathrm{~s}$ \\
\hline & $2^{5}$ & $2.508966 \mathrm{E}-3$ & $2.386773 \mathrm{E}-4$ & 18 & $0.34 \mathrm{~s}$ \\
\hline & $2^{6}$ & $6.569196 \mathrm{E}-4$ & $5.093327 \mathrm{E}-5$ & 23 & $0.78 \mathrm{~s}$ \\
\hline & $2^{7}$ & $1.687507 \mathrm{E}-4$ & $1.019236 \mathrm{E}-5$ & 34 & $4.22 \mathrm{~s}$ \\
\hline & $2^{8}$ & $4.299552 \mathrm{E}-5$ & $2.116632 \mathrm{E}-6$ & 49 & $11.50 \mathrm{~s}$ \\
\hline & $2^{9}$ & $1.086598 \mathrm{E}-5$ & $3.724706 \mathrm{E}-7$ & 72 & $1 \mathrm{~m} 32 \mathrm{~s}$ \\
\hline conv. rate & & $M=50, \kappa=1.95$ & $M=50, \kappa=2.28$ & & \\
\hline \multirow{6}{*}{$\eta=0.1$} & $2^{4}$ & $9.316244 \mathrm{E}-3$ & $4.396209 \mathrm{E}-5$ & 50 & $0.34 \mathrm{~s}$ \\
\hline & $2^{5}$ & $2.482261 \mathrm{E}-3$ & $2.186055 \mathrm{E}-5$ & 82 & $1.30 \mathrm{~s}$ \\
\hline & $2^{6}$ & $6.471300 \mathrm{E}-4$ & $7.664450 \mathrm{E}-6$ & 84 & $3.05 \mathrm{~s}$ \\
\hline & $2^{7}$ & 1.661195E-4 & $2.535404 \mathrm{E}-6$ & 75 & $7.22 \mathrm{~s}$ \\
\hline & $2^{8}$ & $4.232844 \mathrm{E}-5$ & $7.805887 \mathrm{E}-7$ & 77 & $18 \mathrm{~s}$ \\
\hline & $2^{9}$ & $1.074239 \mathrm{E}-5$ & $1.901050 \mathrm{E}-7$ & 83 & $2 \mathrm{~m} \mathrm{29s}$ \\
\hline conv. rate & & $M=50, \kappa=1.95$ & $M=50, \kappa=1.58$ & & \\
\hline \multirow{6}{*}{$\eta=0.01$} & $2^{4}$ & $9.315837 \mathrm{E}-3$ & $4.568987 \mathrm{E}-7$ & 120 & $0.95 \mathrm{~s}$ \\
\hline & $2^{5}$ & $2.480667 \mathrm{E}-3$ & $2.483698 \mathrm{E}-7$ & 961 & $15 \mathrm{~s}$ \\
\hline & $2^{6}$ & $6.461560 \mathrm{E}-4$ & $9.835563 \mathrm{E}-8$ & 330 & $11 \mathrm{~s}$ \\
\hline & $2^{7}$ & $1.656546 \mathrm{E}-4$ & $3.973004 \mathrm{E}-8$ & 380 & $40 \mathrm{~s}$ \\
\hline & $2^{8}$ & $4.213543 \mathrm{E}-5$ & $1.675305 \mathrm{E}-8$ & 447 & $2 \mathrm{~m} \mathrm{1s}$ \\
\hline & $2^{9}$ & $1.068342 \mathrm{E}-5$ & $5.988083 \mathrm{E}-9$ & 522 & $13 \mathrm{~m} 12 \mathrm{~s}$ \\
\hline conv. rate & & $M=50, \kappa=1.96$ & $M=50, \kappa=1.26$ & & \\
\hline
\end{tabular}




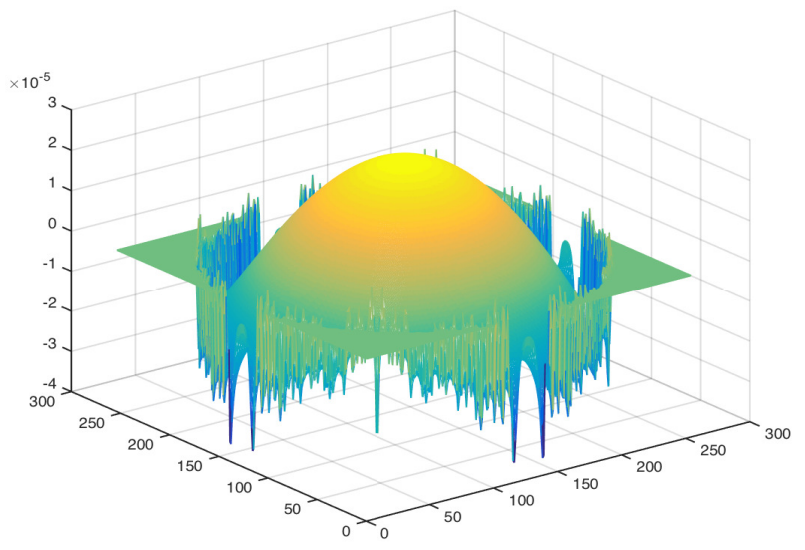

Figure 2: Error of the numerical solution of $\S 8.2$ with $N=2^{8}, \alpha=\beta=0.1$ and $\eta=0.01$.

$O\left(N^{3}\right)$ to approximately linear computational complexity. We then extend the scheme method to conservative fractional diffusion equations defined on a general convex domain, via a volume penalization technique. Numerical experiments were carried out to show the utility of the method.

The fast finite volume scheme is developed for a steady-state spacefractional conservative diffusion equation in a coordinate form, in which the fractional derivatives appear only in the $x$ and $y$ directions. In principle, the extension to a fast finite volume scheme for full space-fractional partial differential equations (in which the fractional derivatives appear in all directions) [10? ] is straightforward. In fast, we have developed a fast finite element scheme for full space-fractional partial differential equations in a rectangular domain with a rectangular partition [9]. However, the derivation is much more involved and will be considered in the near future.

Although we develop a fast finite volume scheme for a steady-state conservative space-fractional diffusion equation in this paper, the extension to time-dependent space-fractional diffusion equations is straightforward as a time-marching procedure can be used. However, the extension to timedependent space-time fractional diffusion equations is much more involved and will be considered in the near future. 


\section{Appendices}

\subsection{Proof of Theorem 1}

We prove the decompositions (10), (12), and (14). To prove (10), we choose $j=l$ in (16) to evaluate the diagonal blocks $A_{l, l}^{x}$ for $l=1,2, \ldots, N_{y}$. We choose $i=k$ in (16) to evaluate the diagonal entries $\left(A_{l, l}^{x}\right)_{k, k}$ for $k=$ $1,2, \ldots, N_{x}$ as follows

$$
\begin{aligned}
\left(A_{l, l}^{x}\right)_{k, k}= & \frac{h_{y} K_{k, l}^{x,-}}{\Gamma(\alpha+1) h_{x}^{1-\alpha}}\left\{\gamma_{x}\left(1+\frac{1}{\alpha+1}\right)\left(\frac{1}{2}\right)^{\alpha+1}\right. \\
& \left.+\left(1-\gamma_{x}\right)\left[\frac{1}{2}\left(2\left(\frac{1}{2}\right)^{\alpha}-\left(\frac{3}{2}\right)^{\alpha}\right)+\frac{1}{\alpha+1}\left(2-\left(\frac{3}{2}\right)^{\alpha+1}\right)\right]\right\} \\
& +\frac{h_{y} K_{k, l}^{x,+}}{\Gamma(\alpha+1) h_{x}^{1-\alpha}}\left\{\gamma _ { x } \left[\frac{1}{2}\left(2\left(\frac{1}{2}\right)^{\alpha}-\left(\frac{3}{2}\right)^{\alpha}\right)\right.\right. \\
& \left.\left.+\frac{1}{\alpha+1}\left(2-\left(\frac{3}{2}\right)^{\alpha+1}\right)\right]+\left(1-\gamma_{x}\right)\left(1+\frac{1}{\alpha+1}\right)\left(\frac{1}{2}\right)^{\alpha+1}\right\} .
\end{aligned}
$$

We choose $i=k-1$ for $k=2, \ldots, N_{x}$ or $i=k+1$ for $k=1, \ldots, N_{x}-1$ in (16) to evaluate the sub- and super-diagonal entries of $A_{l, l}^{x}$ as follows

$$
\begin{aligned}
\left(A_{l, l}^{x}\right)_{k, k-1}= & -\frac{h_{y} K_{k, l}^{x,-}}{\Gamma(\alpha+1) h_{x}^{1-\alpha}}\left\{\gamma _ { x } \left[\frac{1}{2}\left(\left(\frac{1}{2}\right)^{\alpha-1}-\left(\frac{3}{2}\right)^{\alpha}\right)\right.\right. \\
& \left.\left.+\frac{1}{\alpha+1}\left(2-\left(\frac{3}{2}\right)^{\alpha+1}\right)\right]+\left(1-\gamma_{x}\right)\left(1+\frac{1}{\alpha+1}\right)\left(\frac{1}{2}\right)^{\alpha+1}\right] \\
& +\frac{\gamma_{x} h_{y} K_{k, l}^{x,+}}{\Gamma(\alpha+1) h_{x}^{1-\alpha}}\left\{\frac{1}{2}\left[2\left(\frac{3}{2}\right)^{\alpha}-\left(\frac{5}{2}\right)^{\alpha}-\left(\frac{1}{2}\right)^{\alpha}\right]\right. \\
& \left.+\frac{1}{\alpha+1}\left[2^{\alpha+2}-2+\left(\frac{1}{2}\right)^{\alpha+1}-\left(\frac{5}{2}\right)^{\alpha+1}\right]\right\} \\
\left(A_{l, l}^{x}\right)_{k, k+1}= & \frac{\left(1-\gamma_{x}\right) h_{y} K_{k, l}^{x,-}}{\Gamma(\alpha+1) h_{x}^{1-\alpha}}\left\{\frac{1}{2}\left[2\left(\frac{3}{2}\right)^{\alpha}-\left(\frac{5}{2}\right)^{\alpha}-\left(\frac{1}{2}\right)^{\alpha}\right]+\frac{1}{\alpha+1}\left[2^{\alpha+2}-2\right.\right. \\
+ & \left.\left.\left(\frac{1}{2}\right)^{\alpha+1}-\left(\frac{5}{2}\right)^{\alpha+1}\right]\right\}-\frac{h_{y} K_{k, l}^{x,+}}{\Gamma(\alpha+1) h_{x}^{1-\alpha}}\left\{\gamma_{x}\left(1+\frac{1}{\alpha+1}\right)\left(\frac{1}{2}\right)^{\alpha+1}\right. \\
+ & \left.\left(1-\gamma_{x}\right)\left[\frac{1}{2}\left(2\left(\frac{1}{2}\right)^{\alpha}-\left(\frac{3}{2}\right)^{\alpha}\right)+\frac{1}{\alpha+1}\left(2-\left(\frac{3}{2}\right)^{\alpha+1}\right)\right]\right\} .
\end{aligned}
$$


We evaluate the entries outside of the tridiagonals of $A_{l, l}^{x}$ as follows

$$
\begin{aligned}
& \left(A_{l, l}^{x}\right)_{k, i}=\frac{\gamma_{x} h_{y} K_{k, l}^{x,-}}{\Gamma(\alpha+1) h_{x}^{1-\alpha}}\left\{\frac{1}{2}\left[\left(k-i+\frac{1}{2}\right)^{\alpha}-2\left(k-i-\frac{1}{2}\right)^{\alpha}+\left(k-i-\frac{3}{2}\right)^{\alpha}\right]\right. \\
& \left.+\frac{1}{\alpha+1}\left[\left(k-i+\frac{1}{2}\right)^{\alpha+1}-2(k-i)^{\alpha+1}+2(k-i-1)^{\alpha+1}-\left(k-i-\frac{3}{2}\right)^{\alpha+1}\right]\right\} \\
& -\frac{\gamma_{x} h_{y} K_{k, l}^{x,+}}{\Gamma(\alpha+1) h_{x}^{1-\alpha}}\left\{\frac{1}{2}\left[\left(k-i+\frac{3}{2}\right)^{\alpha}-2\left(k-i+\frac{1}{2}\right)^{\alpha}+\left(k-i-\frac{1}{2}\right)^{\alpha}\right]\right. \\
& \left.+\frac{1}{\alpha+1}\left[\left(k-i+\frac{3}{2}\right)^{\alpha+1}-2(k-i+1)^{\alpha+1}+2(k-i)^{\alpha+1}-\left(k-i-\frac{1}{2}\right)^{\alpha+1}\right]\right\} \text {, } \\
& 1 \leq i \leq k-2, \quad 3 \leq k \leq N_{x}, \\
& \left(A_{l, l}^{x}\right)_{k, i}=\frac{\left(1-\gamma_{x}\right) h_{y} K_{k,-}^{x,-}}{\Gamma(\alpha+1) h_{x}^{1-\alpha}}\left\{\frac{1}{2}\left[2\left(i-k+\frac{1}{2}\right)^{\alpha}-\left(i-k+\frac{3}{2}\right)^{\alpha}-\left(i-k-\frac{1}{2}\right)^{\alpha}\right]\right. \\
& \left.+\frac{1}{\alpha+1}\left[\left(i-k-\frac{1}{2}\right)^{\alpha+1}-2(i-k)^{\alpha+1}+2(i-k+1)^{\alpha+1}-\left(i-k+\frac{3}{2}\right)^{\alpha+1}\right]\right\} \\
& -\frac{\left(1-\gamma_{x}\right) h_{y} K_{k, l}^{x,+}}{\Gamma(\alpha+1) h_{x}^{1-\alpha}}\left\{\frac{1}{2}\left[2\left(i-k-\frac{1}{2}\right)^{\alpha}-2\left(i-k+\frac{1}{2}\right)^{\alpha}-\left(i-k-\frac{3}{2}\right)^{\alpha}\right]\right. \\
& \left.+\frac{1}{\alpha+1}\left[\left(i-k-\frac{3}{2}\right)^{\alpha+1}-2(i-k-1)^{\alpha+1}+2(i-k)^{\alpha+1}-\left(i-k+\frac{1}{2}\right)^{\alpha+1}\right]\right\} \\
& k+2 \leq i \leq N_{x}, \quad 1 \leq k \leq N_{x}-2 .
\end{aligned}
$$

We combine the expansions (29)-(30) to finish the proof of (10).

We choose $j=l+1$ in (16) to evaluate the superdiagonal matrix blocks $A_{l, l+1}^{x}$ for $l=1,2, \ldots, N_{y}-1$. We let $i=k$ in (16) to evaluate the diagonal entries $\left(A_{l, l+1}^{x}\right)_{k, k}$ for $k=1,2, \ldots, N_{x}$ as follows

$$
\begin{aligned}
\left(A_{l, l+1}^{x}\right)_{k, k}= & \frac{\left(1-\gamma_{x}\right) h_{y} K_{l, l}^{x,-}}{\Gamma(\alpha+1) h_{x}^{1-\alpha}}\left\{\frac{1}{2}\left[\left(\frac{1}{2}\right)^{\alpha}-\left(\frac{3}{2}\right)^{\alpha}\right]\right. \\
& \left.+\frac{1}{\alpha+1}\left[-1-\left(\frac{1}{2}\right)^{\alpha+1}+\left(\frac{3}{2}\right)^{\alpha+1}\right]\right\} \\
& +\frac{h_{y} K_{k, l}^{x,+}}{\Gamma(\alpha+1) h_{x}^{1-\alpha}}\left\{\gamma_{x}\left[\left(1+\frac{1}{\alpha+1}\right)\left(\frac{1}{2}\right)^{\alpha+1}-\frac{1}{\alpha+1}\right]\right. \\
& \left.+\left(1-\gamma_{x}\right)\left(1-\frac{1}{\alpha+1}\right)\left(\frac{1}{2}\right)^{\alpha+1}\right\} .
\end{aligned}
$$

We then choose $i=k+1, \ldots, N_{x}$ for $k=1, \ldots, N_{x}-1$ in (16) to evaluate 
the upper-triangular entries of $A_{l, l+1}^{x}$ as follows

$$
\begin{aligned}
\left(A_{l, l+1}^{x}\right)_{k, i}= & \frac{\left(1-\gamma_{x}\right) h_{y} K_{k, l}^{x,-}}{\Gamma(\alpha+1) h_{x}^{1-\alpha}}\left\{\frac{1}{2}\left[\left(i-k+\frac{1}{2}\right)^{\alpha}-\left(i-k+\frac{3}{2}\right)^{\alpha}\right]\right. \\
& +\frac{1}{\alpha+1}\left[\left(i-k+\frac{3}{2}\right)^{\alpha+1}-\left(i-k+\frac{1}{2}\right)^{\alpha+1}-(i-k+1)^{\alpha+1}\right. \\
& \left.\left.+(i-k)^{\alpha+1}\right]\right\}-\frac{\left(1-\gamma_{x}\right) h_{y} K_{k, l}^{x,+}}{\Gamma(\alpha+1) h_{x}^{1-\alpha}}\left\{\frac{1}{2}\left[\left(i-k-\frac{1}{2}\right)^{\alpha}-\left(i-k+\frac{1}{2}\right)^{\alpha}\right]\right. \\
& \left.+\frac{1}{\alpha+1}\left[(i-k-1)^{\alpha+1}-\left(i-k-\frac{1}{2}\right)^{\alpha+1}-(i-k)^{\alpha+1}+\left(i-k+\frac{1}{2}\right)^{\alpha+1}\right]\right\} .
\end{aligned}
$$

We next evaluate the subdiagonal entries $\left(A_{l, l+1}^{x}\right)_{k, k-1}$ for $k=2, \ldots, N_{x}$

$$
\begin{aligned}
\left(A_{l, l+1}^{x}\right)_{k, k-1}= & -\frac{h_{y} K_{k, l}^{x,-}}{\Gamma(\alpha+1) h_{x}^{1-\alpha}}\left\{\gamma_{x}\left[\left(1+\frac{1}{\alpha+1}\right)\left(\frac{1}{2}\right)^{\alpha+1}-\frac{1}{\alpha+1}\right]\right. \\
& \left.+\left(1-\gamma_{x}\right)\left(1-\frac{1}{\alpha+1}\right)\left(\frac{1}{2}\right)^{\alpha+1}\right\} \\
& -\frac{\gamma_{x} h_{y} K_{k, l}^{x,+}}{\Gamma(\alpha+1) h_{x}^{1-\alpha}}\left\{\frac{1}{2}\left[\left(\frac{1}{2}\right)^{\alpha}-\left(\frac{3}{2}\right)^{\alpha}\right]\right. \\
& \left.+\frac{1}{\alpha+1}\left[2^{\alpha+1}-1+\left(\frac{1}{2}\right)^{\alpha+1}-\left(\frac{3}{2}\right)^{\alpha+1}\right]\right\} .
\end{aligned}
$$

Finally, we evaluate the lower triangular entries below the subdiagonals of $A_{l, l+1}^{x}$ for $i=1, \ldots, k-2$ and $k=3, \ldots, N_{x}$ as follows

$$
\begin{aligned}
\left(A_{l, l+1}^{x}\right)_{k, i} & :=\frac{\gamma_{x} h_{y} K_{k, l}^{x,-}}{\Gamma(\alpha+1) h_{x}^{1-\alpha}}\left\{\frac{1}{2}\left[\left(k-i-\frac{3}{2}\right)^{\alpha}-\left(k-i-\frac{1}{2}\right)^{\alpha}\right]\right. \\
& +\frac{1}{\alpha+1}\left[(k-i)^{\alpha+1}-(k-i-1)^{\alpha+1}+\left(k-i-\frac{3}{2}\right)^{\alpha+1}\right. \\
& \left.\left.-\left(k-i-\frac{1}{2}\right)^{\alpha+1}\right]\right\}-\frac{\gamma_{x} h_{y} K_{k, l}^{x,+}}{\Gamma(\alpha+1) h_{x}^{1-\alpha}}\left\{\frac { 1 } { 2 } \left[\left(k-i-\frac{1}{2}\right)^{\alpha}\right.\right. \\
& \left.-\left(k-i+\frac{1}{2}\right)^{\alpha}\right]+\frac{1}{\alpha+1}\left[(k-i+1)^{\alpha+1}\right. \\
& \left.\left.-\left(k-i+\frac{1}{2}\right)^{\alpha+1}-(k-i)^{\alpha+1}+\left(k-i-\frac{1}{2}\right)^{\alpha+1}\right]\right\} .
\end{aligned}
$$

We combine expansions (31)-(32) to prove (12). We can similarly choose $j=l-1$ in (16) to evaluate the subdiagonal block matrices $A_{l, l-1}^{x}$ for $l=$ $2, \ldots, N_{y}$ to prove (14) and skip the details. 


\subsection{Proof of Theorem 3}

To prove (19), we evaluate each matrix block $A_{l, j}^{y}$ for $l, j=1,2, \ldots, N_{y}$. We begin with the diagonal matrix blocks $A_{l, l}^{y}$ by choosing $j=l$ in (17) for $l=1,2, \ldots, N_{y}$. We evaluate their diagonal entries $\left(A_{l, l}^{y}\right)_{k, k}$ by further choosing $i=k$ in (17) for $k=1,2, \ldots, N_{x}$ to obtain

$$
\begin{aligned}
\left(A_{l, l}^{y}\right)_{k, k}= & \frac{h_{x} K_{k, l}^{y,-}}{\Gamma(\beta+1) h_{y}^{1-\beta}}\left\{\gamma_{y}\left(1+\frac{1}{\beta+1}\right)\left(\frac{1}{2}\right)^{\beta+1}+\left(1-\gamma_{y}\right)\left[\frac{1}{2}\left(\left(\frac{1}{2}\right)^{\beta-1}-\left(\frac{3}{2}\right)^{\beta}\right)\right.\right. \\
& \left.+\frac{1}{\beta+1}\left[2-\left(\frac{3}{2}\right)^{\beta+1}\right]\right\}+\frac{h_{x} K_{k, l}^{y,+}}{\Gamma(\beta+1) h_{y}^{1-\beta}}\left\{\gamma _ { y } \left[\frac{1}{2}\left(2\left(\frac{1}{2}\right)^{\beta}-\left(\frac{3}{2}\right)^{\beta}\right)\right.\right. \\
& \left.\left.+\frac{1}{\beta+1}\left(2-\left(\frac{3}{2}\right)^{\beta+1}\right)\right]+\left(1-\gamma_{y}\right)\left(1+\frac{1}{\beta+1}\right)\left(\frac{1}{2}\right)^{\beta+1}\right\} .
\end{aligned}
$$

We then choose $i=k-1$ in (17) for $k=2,3, \ldots, N_{y}$ and $i=k+1$ in (17) for $k=1,2, \ldots, N_{y}-1$, respectively, to evaluate the subdiagonal and superdiagonal entries of $A_{l, l}^{y}$

$$
\begin{aligned}
\left(A_{l, l}^{y}\right)_{k, k-1}= & \frac{h_{x} K_{k, l}^{y,-}}{\Gamma(\beta+1) h_{y}^{1-\beta}}\left\{\gamma_{y}\left(1-\frac{1}{\beta+1}\right)\left(\frac{1}{2}\right)^{\beta+1}+\left(1-\gamma_{y}\right)\left[\left(1+\frac{1}{\beta+1}\right)\left(\frac{1}{2}\right)^{\beta+1}\right.\right. \\
& \left.\left.-\frac{1}{\beta+1}\right]\right\}+\frac{\gamma_{y} h_{x} K_{k, l}^{y,+}}{\Gamma(\beta+1) h_{y}^{1-\beta}}\left\{\frac{1}{2}\left[\left(\frac{1}{2}\right)^{\beta}-\left(\frac{3}{2}\right)^{\beta}\right]\right. \\
& \left.+\frac{1}{\beta+1}\left[\left(\frac{3}{2}\right)^{\beta+1}-\left(\frac{1}{2}\right)^{\beta+1}-1\right]\right\}, \\
\left(A_{l, l}^{y}\right)_{k, k+1}= & \frac{\left(1-\gamma_{y}\right) h_{x} K_{k, l}^{y,-}}{\Gamma(\beta+1) h_{y}^{1-\beta}}\left\{\frac{1}{2}\left[\left(\frac{1}{2}\right)^{\beta}-\left(\frac{3}{2}\right)^{\beta}\right]+\frac{1}{\beta+1}\left[\left(\frac{3}{2}\right)^{\beta+1}-\left(\frac{1}{2}\right)^{\beta+1}-1\right]\right\} \\
& +\frac{h_{x} K_{k, l}^{y,+}}{\Gamma(\beta+1) h_{y}^{1-\beta}}\left\{\gamma_{y}\left[\left(1+\frac{1}{\beta+1}\right)\left(\frac{1}{2}\right)^{\beta+1}-\frac{1}{\beta+1}\right]\right. \\
& \left.+\left(1-\gamma_{y}\right)\left(1-\frac{1}{\beta+1}\right)\left(\frac{1}{2}\right)^{\beta+1}\right\} .
\end{aligned}
$$

We combine (21) with the three preceding equations to finish the evaluation of $A_{l, l}^{y}$ for $l=1,2, \ldots, N_{y}$.

We next evaluate the superdiagonal matrix blocks $A_{l, l+1}^{y}$ for $l=1,2, \ldots, N_{y}-$

1. We begin by evaluating their diagonal entries by choosing $i=k$ in (17) 
for $k=1,2, \ldots, N_{x}$

$$
\begin{aligned}
\left(A_{l, l+1}\right)_{k, k}= & \frac{\left(1-\gamma_{y}\right) h_{x} K_{k, l}^{y,-}}{\Gamma(\beta+1) h_{y}^{1-\beta}}\left\{\frac{1}{2}\left[2\left(\frac{3}{2}\right)^{\beta}-\left(\frac{5}{2}\right)^{\beta}-\left(\frac{1}{2}\right)^{\beta}\right]+\frac{1}{\beta+1}\left[2^{\beta+2}-2\right.\right. \\
& \left.\left.+\left(\frac{1}{2}\right)^{\beta+1}-\left(\frac{5}{2}\right)^{\beta+1}\right]\right\}-\frac{h_{x} K_{k, l}^{y,+}}{\Gamma(\beta+1) h_{y}^{1-\beta}}\left\{\gamma_{y}\left(1+\frac{1}{\beta+1}\right)\left(\frac{1}{2}\right)^{\beta+1}\right. \\
& \left.+\left(1-\gamma_{y}\right)\left[\frac{1}{2}\left(\left(\frac{1}{2}\right)^{\beta-1}-\left(\frac{3}{2}\right)^{\beta}\right)+\frac{1}{\beta+1}\left(2-\left(\frac{3}{2}\right)^{\beta+1}\right)\right]\right\} .
\end{aligned}
$$

We then evaluate the subdiagonals and subdiagonals of $A_{l, l+1}^{y}$ for $l=$ $1,2, \ldots, N_{y}-1$ as follows

$$
\begin{aligned}
& \left(A_{l, l+1}\right)_{k, k-1}=-\frac{\left(1-\gamma_{y}\right) h_{x} K_{k, l}^{x,-}}{\Gamma(\beta+1) h_{y}^{1-\beta}}\left\{\frac{1}{2}\left[\left(\frac{1}{2}\right)^{\beta}-\left(\frac{3}{2}\right)^{\beta}\right]+\frac{1}{\beta+1}\left[2^{\beta+1}-1\right.\right. \\
& \left.\left.+\left(\frac{1}{2}\right)^{\beta+1}-\left(\frac{3}{2}\right)^{\beta+1}\right]\right\}-\frac{h_{x} K_{k, l}^{y,+}}{\Gamma(\beta+1) h_{y}^{1-\beta}}\left\{\gamma_{y}\left(1-\frac{1}{\beta+1}\right)\left(\frac{1}{2}\right)^{\beta+1}\right. \\
& \left.+\left(1-\gamma_{y}\right)\left[\left(1+\frac{1}{\beta+1}\right)\left(\frac{1}{2}\right)^{\beta+1}-\frac{1}{\beta+1}\right]\right\} \text {, } \\
& \left(A_{l, l+1}\right)_{k, k+1}=\frac{\left(1-\gamma_{y}\right) h_{x} K_{k, l}^{x,-}}{\Gamma(\beta+1) h_{y}^{1-\beta}}\left\{\frac{1}{2}\left[\left(\frac{3}{2}\right)^{\beta}-\left(\frac{5}{2}\right)^{\beta}\right]-\frac{1}{\beta+1}\left[2^{\beta+1}-1\right.\right. \\
& \left.\left.+\left(\frac{3}{2}\right)^{\beta+1}-\left(\frac{5}{2}\right)^{\beta+1}\right]\right\}-\frac{\left(1-\gamma_{y}\right) h_{x} K_{k, l}^{x,+}}{\Gamma(\beta+1) h_{y}^{1-\beta}}\left\{\frac{1}{2}\left[\left(\frac{1}{2}\right)^{\beta}-\left(\frac{3}{2}\right)^{\beta}\right]\right. \\
& \left.+\frac{1}{\beta+1}\left[\left(\frac{3}{2}\right)^{\beta+1}-\left(\frac{1}{2}\right)^{\beta+1}-1\right]\right\} \text {. }
\end{aligned}
$$

We combine (21) with equation (33) and the two following equations to finish the evaluation of the superdiagonal matrix blocks $A_{l, l+1}^{y}$ for $l=1,2, \ldots, N_{y}-$ 1.

We now turn to the upper triangular matrix blocks above the superdiagonal, i.e., $\left(A_{l, j}^{y}\right)$ for $l+2 \leq j \leq N_{y}$ with $l=1,2, \cdots, N_{y}-2$. We evaluate 
their tridiagonal entries as follows

$$
\begin{aligned}
& \left(A_{l, j}^{x}\right)_{k, k}=\frac{\left(1-\gamma_{y}\right) h_{x} K_{k, l}^{y,-}}{\Gamma(\beta+1) h_{y}^{1-\beta}}\left\{\frac{1}{2}\left[2\left(j-l+\frac{1}{2}\right)^{\beta}-\left(j-l+\frac{3}{2}\right)^{\beta}-\left(j-l-\frac{1}{2}\right)^{\beta}\right]\right. \\
& \left.+\frac{1}{\beta+1}\left[\left(j-l-\frac{1}{2}\right)^{\beta+1}-2(j-l)^{\beta+1}+2(j-l+1)^{\beta+1}-\left(j-l+\frac{3}{2}\right)^{\beta+1}\right]\right\} \\
& -\frac{\left(1-\gamma_{y}\right) h_{x} K_{k, l}^{y,+}}{\Gamma(\beta+1) h_{y}^{1-\beta}}\left\{\frac{1}{2}\left[2\left(j-l-\frac{1}{2}\right)^{\beta}-\left(j-l+\frac{1}{2}\right)^{\beta}-\left(j-l-\frac{3}{2}\right)^{\beta}\right]\right. \\
& \left.+\frac{1}{\beta+1}\left[\left(j-l-\frac{3}{2}\right)^{\beta+1}-2(j-l-1)^{\beta+1}+2(j-l)^{\beta+1}-\left(j-l+\frac{1}{2}\right)^{\beta+1}\right]\right\} \text {, } \\
& \left(A_{l, j}^{y}\right)_{k, k-1}=\frac{\left(1-\gamma_{y}\right) h_{x} K_{k,-}^{y,-}}{\Gamma(\beta+1) h_{y}^{1-\beta}}\left\{\frac{1}{2}\left[\left(j-l+\frac{1}{2}\right)^{\beta}-\left(j-l-\frac{1}{2}\right)^{\beta}\right]\right. \\
& -\frac{1}{\beta+1}\left[\left(j-l-\frac{1}{2}\right)^{\beta+1}-(j-l)^{\beta+1}-\left(j-l+\frac{1}{2}\right)^{\beta+1}\right. \\
& \left.\left.+(j-l+1)^{\beta+1}\right]\right\}-\frac{\left(1-\gamma_{y}\right) h_{x} K_{k, l}^{x,+}}{\Gamma(\beta+1) h_{y}^{1-\beta}}\left\{\frac { 1 } { 2 } \left[\left(j-l-\frac{1}{2}\right)^{\beta}\right.\right. \\
& \left.-\left(j-l-\frac{3}{2}\right)^{\beta}\right]-\frac{1}{\beta+1}\left[\left(j-l-\frac{3}{2}\right)^{\beta+1}-(j-l-1)^{\beta+1}\right. \\
& \left.\left.-\left(j-l-\frac{1}{2}\right)^{\beta+1}+(j-l)^{\beta+1}\right]\right\} \text {, } \\
& \left(A_{l, j}^{y}\right)_{k, k+1}=\frac{\left(1-\gamma_{y}\right) h_{x} K_{k, l}^{x,-}}{\Gamma(\beta+1) h_{y}^{1-\beta}}\left\{\frac{1}{2}\left[\left(j-l+\frac{1}{2}\right)^{\beta}-\left(j-l+\frac{3}{2}\right)^{\beta}\right]\right. \\
& +\frac{1}{\alpha+1}\left[(j-l)^{\alpha+1}-\left(j-l+\frac{1}{2}\right)^{\beta+1}-(j-l+1)^{\beta+1}\right. \\
& \left.\left.+\left(j-l+\frac{3}{2}\right)^{\beta+1}\right]\right\}-\frac{\left(1-\gamma_{y}\right) h_{x} K_{k, l}^{y,+}}{\Gamma(\beta+1) h_{y}^{1-\beta}}\left\{\frac { 1 } { 2 } \left[\left(j-l-\frac{1}{2}\right)^{\beta}\right.\right. \\
& \left.-\left(j-l+\frac{1}{2}\right)^{\beta}\right]+\frac{1}{\beta+1}\left[(j-l-1)^{\beta+1}-\left(j-l-\frac{1}{2}\right)^{\beta+1}\right. \\
& \left.\left.-(j-l)^{\beta+1}+\left(j-l+\frac{1}{2}\right)^{\beta+1}\right]\right\} \text {. }
\end{aligned}
$$

We combine (21) and (34)-(35) to finish the evaluation of matrix blocks $A_{l, j}^{y}$ for $l+2 \leq j \leq N_{y}$ with $l=1,2, \cdots, N_{y}-2$.

We similarly evaluate the subdiagonal matrix blocks $A_{l, l-1}^{y}$ for $l=2,3, \ldots, N_{y}$ 
as follows

$$
\begin{aligned}
&\left(A_{l, l-1}^{y}\right)_{k, k}=-\frac{h_{x} K_{k, j}^{y,-}}{\Gamma(\beta+1) h_{y}^{1-\beta}}\left\{\gamma_{y}\left[\frac{1}{2}\left(\left(\frac{1}{2}\right)^{\beta-1}-\left(\frac{3}{2}\right)^{\beta}\right)+\frac{1}{\beta+1}\left(2-\left(\frac{3}{2}\right)^{\beta+1}\right)\right]\right. \\
&+\left.\left(1-\gamma_{y}\right)\left(1+\frac{1}{\beta+1}\right)\left(\frac{1}{2}\right)^{\beta+1}\right]+\frac{\gamma_{y} h_{x} K_{k, l}^{y,+}}{\Gamma(\beta+1) h_{y}^{1-\beta}}\left\{\frac { 1 } { 2 } \left[2\left(\frac{3}{2}\right)^{\beta}-\left(\frac{1}{2}\right)^{\beta}\right.\right. \\
&\left.\left.-\left(\frac{5}{2}\right)^{\beta}\right]+\frac{1}{\beta+1}\left[2^{\beta+2}-2+\left(\frac{1}{2}\right)^{\beta+1}-\left(\frac{5}{2}\right)^{\beta+1}\right]\right\} \\
&\left(A_{l, l-1}^{y}\right)_{k, k-1}=\frac{\gamma_{y} h_{x} K_{k, l}^{y,-}}{\Gamma(\beta+1) h_{y}^{1-\beta}}\left\{\frac{1}{2}\left[\left(\frac{3}{2}\right)^{\beta}-\left(\frac{1}{2}\right)^{\beta}\right]-\frac{1}{\beta+1}\left[\left(\frac{3}{2}\right)^{\beta+1}\right.\right. \\
&\left.\left.-1-\left(\frac{1}{2}\right)^{\beta+1}\right]\right\}-\frac{\gamma_{y} h_{x} K_{k, l}^{y,+}}{\Gamma(\beta+1) h_{y}^{1-\beta}}\left\{\frac{1}{2}\left[\left(\frac{5}{2}\right)^{\beta}-\left(\frac{3}{2}\right)^{\beta}\right]\right. \\
&\left.-\frac{1}{\beta+1}\left[\left(\frac{5}{2}\right)^{\beta+1}-2^{\beta+1}-\left(\frac{3}{2}\right)^{\beta+1}+1\right]\right\} \\
&\left(A_{l, l-1}^{y}\right)_{k, k+1}=-\frac{h_{x} K_{k, l}^{y,-}}{\Gamma(\beta+1) h_{y}^{1-\alpha}}\left\{\gamma_{y}\left[\left(1+\frac{1}{\beta+1}\right)\left(\frac{1}{2}\right)^{\beta+1}-\frac{1}{\beta+1}\right]\right. \\
&\left.+\left(1-\gamma_{y}\right)\left(1-\frac{1}{\beta+1}\right)\left(\frac{1}{2}\right)^{\beta+1}\right]+\frac{\gamma_{y} h_{x} K_{k, l}^{y,+}}{\Gamma(\beta+1) h_{y}^{1-\alpha}}\left\{\frac { 1 } { 2 } \left[\left(\frac{1}{2}\right)^{\beta}\right.\right. \\
&\left.\left.-\left(\frac{3}{2}\right)^{\beta}\right]-\frac{1}{\beta+1}\left[2^{\beta+1}-1+\left(\frac{1}{2}\right)^{\beta+1}-\left(\frac{3}{2}\right)^{\beta+1}\right]\right\} .
\end{aligned}
$$

We combine the preceding three equations with (21) to finish the evaluation.

We finally evaluate the lower triangular matrix blocks below the subdiagonal, i.e., $A_{l, j}^{y}$ for $1 \leq j \leq l-2$ with $l=3,4, \cdots, N_{y}$ as follows

$$
\begin{aligned}
\left(A_{l, j}^{y}\right)_{k, k}= & \frac{\gamma_{y} h_{x} K_{k, l}^{y,-}}{\Gamma(\beta+1) h_{y}^{1-\alpha}}\left\{\frac{1}{2}\left[\left(l-j+\frac{1}{2}\right)^{\beta}-2\left(l-j-\frac{1}{2}\right)^{\beta}+\left(l-j-\frac{3}{2}\right)^{\beta}\right]\right. \\
& +\frac{1}{\beta+1}\left[\left(l-j+\frac{1}{2}\right)^{\beta+1}-2(l-j)^{\beta+1}+2(l-j-1)^{\beta+1}\right. \\
& \left.\left.-\left(l-j-\frac{3}{2}\right)^{\beta+1}\right]\right\}-\frac{\gamma_{y} h_{x} K_{k, l}^{y,+}}{\Gamma(\beta+1) h_{y}^{1-\beta}}\left\{\frac { 1 } { 2 } \left[\left(l-j+\frac{3}{2}\right)^{\beta}\right.\right. \\
& \left.-2\left(l-j+\frac{1}{2}\right)^{\beta}+\left(l-j-\frac{1}{2}\right)^{\beta}\right]+\frac{1}{\beta+1}\left[\left(l-j+\frac{3}{2}\right)^{\beta+1}\right. \\
& \left.\left.-2(l-i+1)^{\beta+1}+2(l-j)^{\beta+1}-\left(l-j-\frac{1}{2}\right)^{\beta+1}\right]\right\},
\end{aligned}
$$




$$
\begin{aligned}
& \left(A_{l, j}^{y}\right)_{k, k-1}=\frac{\gamma_{y} h_{x} K_{k, l}^{y,-}}{\Gamma(\beta+1) h_{y}^{1-\beta}}\left\{\frac{1}{2}\left[\left(l-j+\frac{1}{2}\right)^{\beta}-\left(l-j-\frac{1}{2}\right)^{\beta}\right]\right. \\
& -\frac{1}{\beta+1}\left[\left(l-j+\frac{1}{2}\right)^{\beta+1}-(l-j)^{\beta+1}-\left(l-j-\frac{1}{2}\right)^{\beta+1}\right. \\
& \left.\left.+(l-j-1)^{\beta+1}\right]\right\}-\frac{\gamma_{y} h_{x} K_{k, l}^{y,+}}{\Gamma(\beta+1) h_{y}^{1-\beta}}\left\{\frac { 1 } { 2 } \left[\left(l-j+\frac{3}{2}\right)^{\beta}\right.\right. \\
& \left.-\left(l-j+\frac{1}{2}\right)^{\beta}\right]-\frac{1}{\beta+1}\left[\left(l-j+\frac{3}{2}\right)^{\beta+1}-(l-j+1)^{\beta+1}\right. \\
& \left.\left.-\left(l-j+\frac{1}{2}\right)^{\beta+1}+(l-j)^{\alpha+1}\right]\right\} \text {, } \\
& \left(A_{l, j}^{y}\right)_{k, k+1}=\frac{\gamma_{y} h_{x} K_{k, l}^{y,-}}{\Gamma(\beta+1) h_{y}^{1-\beta}}\left\{\frac{1}{2}\left[\left(l-j-\frac{3}{2}\right)^{\beta}-\left(l-j-\frac{1}{2}\right)^{\beta}\right]\right. \\
& +\frac{1}{\beta+1}\left[(l-j)^{\beta+1}-\left(l-j-\frac{1}{2}\right)^{\beta+1}-(l-j-1)^{\beta+1}\right. \\
& \left.\left.+\left(l-j-\frac{3}{2}\right)^{\beta+1}\right]\right\}-\frac{\gamma_{y} h_{x} K_{k, l}^{y,+}}{\Gamma(\beta+1) h_{y}^{1-\beta}}\left\{\frac { 1 } { 2 } \left[\left(l-j-\frac{1}{2}\right)^{\beta}\right.\right. \\
& \left.-\left(l-j+\frac{1}{2}\right)^{\beta}\right]+\frac{1}{\beta+1}\left[(l-j+1)^{\beta+1}-\left(l-j+\frac{1}{2}\right)^{\beta+1}\right. \\
& \left.\left.-(l-j)^{\beta+1}+\left(l-j-\frac{1}{2}\right)^{\beta+1}\right]\right\} \text {. }
\end{aligned}
$$

A direct inspection of the expressions of all the matrix blocks finishes the proof of (19).

\subsection{Expression of the right-hand side in $\S 8.2$}

Let $\Omega_{s}$ be the unit disk centered at the origin and $\Omega_{i}, i=1,2,3,4$, be the lower left, lower right, upper left, and upper right parts of the domain $(-1,1)^{2} \backslash \Omega_{s}$. Let $x_{c}=\sqrt{1-y^{2}}$ and $y_{c}=\sqrt{1-x^{2}}$. Then the right-hand side $f$ in the example in $\S 8.2$ is defined to be $f(x, y)=f_{s}(x, y)$ for $(x, y) \in \Omega_{s}$ 
and $f(x, y)=f_{i}(x, y)$ for $(x, y) \in \Omega_{i}$ with $i=1,2,3,4$. Here

$$
\begin{aligned}
f_{s}(x, y)= & \frac{2\left(1-y^{2}\right)}{\Gamma(\alpha)}\left[\frac{1}{\alpha}\left(\left(x_{c}-x\right)^{\alpha}+\left(x_{c}+x\right)^{\alpha}\right)-x_{c}\left(\left(x_{c}-x\right)^{\alpha-1}+\left(x_{c}+x\right)^{\alpha-1}\right)\right] \\
& +\frac{2\left(1-x^{2}\right)}{\Gamma(\beta)}\left[\frac{1}{\beta}\left(\left(y_{c}-y\right)^{\beta}+\left(y_{c}+y\right)^{\beta}\right)-y_{c}\left(\left(y_{c}-y\right)^{\beta-1}+\left(y_{c}+y\right)^{\beta-1}\right)\right] \\
& +2\left[\frac{x_{c}^{3}}{\Gamma(\alpha)}\left(\left(x_{c}+x\right)^{\alpha-1}+\left(x_{c}-x\right)^{\alpha-1}\right)-\frac{3 x_{c}^{2}}{\Gamma(\alpha+1)}\left(\left(x_{c}+x\right)^{\alpha}+\left(x_{c}-x\right)^{\alpha}\right)\right. \\
& \left.+\frac{6 x_{c}}{\Gamma(\alpha+2)}\left(\left(x_{c}+x\right)^{\alpha+1}+\left(x_{c}-x\right)^{\alpha+1}\right)-\frac{6}{\Gamma(\alpha+3)}\left(\left(x_{c}+x\right)^{\alpha+2}+\left(x_{c}-x\right)^{\alpha+2}\right)\right] \\
& +2\left[\frac{y_{c}^{3}}{\Gamma(\beta)}\left(\left(y_{c}+y\right)^{\beta-1}+\left(y_{c}-y\right)^{\beta-1}\right)-\frac{3 y_{c}^{2}}{\Gamma(\beta+1)}\left(\left(y_{c}+y\right)^{\beta}+\left(y_{c}-y\right)^{\beta}\right)\right. \\
& \left.+\frac{6 y_{c}}{\Gamma(\beta+2)}\left(\left(y_{c}+y\right)^{\beta+1}+\left(y_{c}-y\right)^{\beta+1}\right)-\frac{6}{\Gamma(\beta+3)}\left(\left(y_{c}+y\right)^{\beta+2}+\left(y_{c}-y\right)^{\beta+2}\right)\right], \\
f_{1}(x, y)= & \frac{2\left(1-y^{2}\right)}{\Gamma(\alpha)}\left[\frac{1}{\alpha}\left(\left(x_{c}-x\right)^{\alpha}-\left(-x_{c}-x\right)^{\alpha}\right)-x_{c}\left(\left(x_{c}-x\right)^{\alpha-1}+\left(-x_{c}-x\right)^{\alpha-1}\right)\right] \\
& +2\left(1-x^{2}\right) \frac{1}{\Gamma(\beta)}\left[\frac{1}{\beta}\left(\left(y_{c}-y\right)^{\beta}-\left(-y_{c}-y\right)^{\beta}\right)-y_{c}\left(\left(y_{c}-y\right)^{\beta-1}+\left(-y_{c}-y\right)^{\beta-1}\right)\right. \\
& +2\left[\frac{x_{c}^{3}}{\Gamma(\alpha)}\left(\left(x_{c}-x\right)^{\alpha-1}+\left(-x_{c}-x\right)^{\alpha-1}\right)-\frac{3 x_{c}^{2}}{\Gamma(\alpha+1)}\left(\left(x_{c}-x\right)^{\alpha}-\left(-x_{c}-x\right)^{\alpha}\right)\right. \\
& \left.+\frac{6 x_{c}}{\Gamma(\alpha+2)}\left(\left(x_{c}-x\right)^{\alpha+1}+\left(-x_{c}-x\right)^{\alpha+1}\right)-\frac{6}{\Gamma(\alpha+3)}\left(\left(x_{c}-x\right)^{\alpha+2}-\left(-x_{c}-x\right)^{\alpha+2}\right)\right] \\
& +2\left[\frac{y_{c}^{3}}{\Gamma(\beta)}\left(\left(y_{c}-y\right)^{\beta-1}+\left(-y_{c}-y\right)^{\beta-1}\right)-\frac{3 y_{c}^{2}}{\Gamma(\beta+1)}\left(\left(y_{c}-y\right)^{\beta}-\left(-y_{c}-y\right)^{\beta}\right)\right. \\
& \left.+\frac{6 y_{c}}{\Gamma(\beta+2)}\left(\left(y_{c}-y\right)^{\beta+1}+\left(-y_{c}-y\right)^{\beta+1}\right)-\frac{6}{\Gamma(\beta+3)}\left(\left(y_{c}-y\right)^{\beta+2}-\left(-y_{c}-y\right)^{\beta+2}\right)\right],
\end{aligned}
$$




$$
\begin{aligned}
& f_{2}(x, y)=\frac{2\left(1-y^{2}\right)}{\Gamma(\alpha)}\left[-\frac{1}{\alpha}\left(\left(x-x_{c}\right)^{\alpha}-\left(x_{c}+x\right)^{\alpha}\right)-x_{c}\left(\left(x-x_{c}\right)^{\alpha-1}+\left(x_{c}+x\right)^{\alpha-1}\right)\right] \\
& +\frac{2\left(1-x^{2}\right)}{\Gamma(\beta)}\left[\frac{1}{\beta}\left(\left(y_{c}-y\right)^{\beta}-\left(-y_{c}-y\right)^{\beta}\right)-y_{c}\left(\left(y_{c}-y\right)^{\beta-1}+\left(-y_{c}-y\right)^{\beta-1}\right)\right] \\
& +2\left[\frac{x_{c}^{3}}{\Gamma(\alpha)}\left(\left(x-x_{c}\right)^{\alpha-1}+\left(x+x_{c}\right)^{\alpha-1}\right)+\frac{3 x_{c}^{2}}{\Gamma(\alpha+1)}\left(\left(x-x_{c}\right)^{\alpha}-\left(x+x_{c}\right)^{\alpha}\right)\right. \\
& \left.+\frac{6 x_{c}}{\Gamma(\alpha+2)}\left(\left(x-x_{c}\right)^{\alpha+1}+\left(x+x_{c}\right)^{\alpha+1}\right)+\frac{6}{\Gamma(\alpha+3)}\left(\left(x-x_{c}\right)^{\alpha+2}-\left(x+x_{c}\right)^{\alpha+2}\right)\right] \\
& +2\left[\frac{y_{c}^{3}}{\Gamma(\beta)}\left(\left(y_{c}-y\right)^{\beta-1}+\left(-y_{c}-y\right)^{\beta-1}\right)-\frac{3 y_{c}^{2}}{\Gamma(\beta+1)}\left(\left(y_{c}-y\right)^{\beta}-\left(-y_{c}-y\right)^{\beta}\right)\right. \\
& \left.+\frac{6 y_{c}}{\Gamma(\beta+2)}\left(\left(y_{c}-y\right)^{\beta+1}+\left(-y_{c}-y\right)^{\beta+1}\right)-\frac{6}{\Gamma(\beta+3)}\left(\left(y_{c}-y\right)^{\beta+2}-\left(-y_{c}-y\right)^{\beta+2}\right)\right] \text {, } \\
& f_{3}(x, y)=\frac{2\left(1-y^{2}\right)}{\Gamma(\alpha)}\left[\frac{1}{\alpha}\left(\left(x_{c}-x\right)^{\alpha}-\left(-x_{c}-x\right)^{\alpha}\right)-x_{c}\left(\left(x_{c}-x\right)^{\alpha-1}+\left(-x_{c}-x\right)^{\alpha-1}\right)\right] \\
& -\frac{2\left(1-x^{2}\right)}{\Gamma(\beta)}\left[\frac{1}{\beta}\left(\left(y-y_{c}\right)^{\beta}-\left(y_{c}+y\right)^{\beta}\right)+y_{c}\left(\left(y-y_{c}\right)^{\beta-1}+\left(y_{c}+y\right)^{\beta-1}\right)\right] \\
& +2\left[\frac{x_{c}^{3}}{\Gamma(\alpha)}\left(\left(x-x_{c}\right)^{\alpha-1}+\left(x+x_{c}\right)^{\alpha-1}\right)+\frac{3 x_{c}^{2}}{\Gamma(\alpha+1)}\left(\left(x-x_{c}\right)^{\alpha}-\left(x+x_{c}\right)^{\alpha}\right)\right. \\
& \left.+\frac{6 x_{c}}{\Gamma(\alpha+2)}\left(\left(x-x_{c}\right)^{\alpha+1}+\left(x+x_{c}\right)^{\alpha+1}\right)+\frac{6}{\Gamma(\alpha+3)}\left(\left(x-x_{c}\right)^{\alpha+2}-\left(x+x_{c}\right)^{\alpha+2}\right)\right] \\
& +2\left[\frac{y_{c}^{3}}{\Gamma(\beta)}\left(\left(y-y_{c}\right)^{\beta-1}+\left(y+y_{c}\right)^{\beta-1}\right)+\frac{3 y_{c}^{2}}{\Gamma(\beta+1)}\left(\left(y-y_{c}\right)^{\beta}-\left(y+y_{c}\right)^{\beta}\right)\right. \\
& \left.+\frac{6 y_{c}}{\Gamma(\beta+2)}\left(\left(y-y_{c}\right)^{\beta+1}+\left(y+y_{c}\right)^{\beta+1}\right)+\frac{6}{\Gamma(\beta+3)}\left(\left(y-y_{c}\right)^{\beta+2}-\left(y+y_{c}\right)^{\beta+2}\right)\right] \text {, }
\end{aligned}
$$




$$
\begin{aligned}
f_{4}(x, y) & =\frac{2\left(1-y^{2}\right)}{\Gamma(\alpha)}\left[-\frac{1}{\alpha}\left(\left(x-x_{c}\right)^{\alpha}-\left(x_{c}+x\right)^{\alpha}\right)-x_{c}\left(\left(x-x_{c}\right)^{\alpha-1}+\left(x_{c}+x\right)^{\alpha-1}\right)\right] \\
& +\frac{2\left(1-x^{2}\right)}{\Gamma(\beta)}\left[-\frac{1}{\beta}\left(\left(y-y_{c}\right)^{\beta}-\left(y_{c}+y\right)^{\beta}\right)-y_{c}\left(\left(y-y_{c}\right)^{\beta-1}+\left(y_{c}+y\right)^{\beta-1}\right)\right] \\
& +2\left[\frac{x_{c}^{3}}{\Gamma(\alpha)}\left(\left(x-x_{c}\right)^{\alpha-1}+\left(x+x_{c}\right)^{\alpha-1}\right)+\frac{3 x_{c}^{2}}{\Gamma(\alpha+1)}\left(\left(x-x_{c}\right)^{\alpha}-\left(x+x_{c}\right)^{\alpha}\right)\right. \\
& \left.+\frac{6 x_{c}}{\Gamma(\alpha+2)}\left(\left(x-x_{c}\right)^{\alpha+1}+\left(x+x_{c}\right)^{\alpha+1}\right)+\frac{6}{\Gamma(\alpha+3)}\left(\left(x-x_{c}\right)^{\alpha+2}-\left(x+x_{c}\right)^{\alpha+2}\right)\right] \\
& +2\left[\frac{y_{c}^{3}}{\Gamma(\beta)}\left(\left(y-y_{c}\right)^{\beta-1}+\left(y+y_{c}\right)^{\beta-1}\right)+\frac{3 y_{c}^{2}}{\Gamma(\beta+1)}\left(\left(y-y_{c}\right)^{\beta}-\left(y+y_{c}\right)^{\beta}\right)\right. \\
& \left.+\frac{6 y_{c}}{\Gamma(\beta+2)}\left(\left(y-y_{c}\right)^{\beta+1}+\left(y+y_{c}\right)^{\beta+1}\right)+\frac{6}{\Gamma(\beta+3)}\left(\left(y-y_{c}\right)^{\beta+2}-\left(y+y_{c}\right)^{\beta+2}\right)\right] .
\end{aligned}
$$

\section{Acknowledgements}

This work was supported in part by the National Science Foundation under Grants EAR-0934747 and DMS-1216923, by the OSD/ARO MURI Grant W911NF-15-1-0562, by the National Natural Science Foundation of China under Grants 91130010, 11471194, and 11571115, and by the State Scholarship Fund from China Scholarship Council under Grant 201306220110. The authors would like to express their sincere thanks to the referees for their very helpful comments and suggestions, which greatly improved the quality of this paper.

\section{References}

[1] P. Angot, C.-H. Bruneau, and P. Fabrie, A penalization method to take into account obstacles in incompressible visocous flows, Numer. Math., 81 (1999), 491-520.

[2] M. Bebendorf, Hierarchical Matrices, Springer, New York, 2008.

[3] D. Benson, S.W. Wheatcraft, and M.M. Meerschaert, The fractionalorder governing equation of Lévy motion, Water Resour. Res. 36 (2000), 1413-1423. 
[4] G. Carbou and P. Fabrie, Boundary layer for a penalization method for viscous impcompressible flow, Adv. Diff. Eqn., 8 (2003), 1453-1480.

[5] R.H. Chan and M.K. Ng, Conjugate gradient methods for Toeplitz systems, SIAM Review, 38 (1996), 427-482.

[6] T. Chan, An optimal circulant preconditioner for Toeplitz systems, SIAM J. Sci. Stat. Comput., 9 (1988), 766-771.

[7] P.J. Davis, Circulant Matrices, Wiley-Intersciences, New York, 1979.

[8] D. del-Castillo-Negrete, B.A. Carreras, and V. E. Lynch, Fractional diffusion in plasma turbulence, Phys. Plasmas, 11 (2004), 3854.

[9] N. Du and H. Wang, A fast finite element method for space-fractional dispersion equations on bounded domains in $\mathbb{R}^{2}$, SIAM J. Sci. Comput., 37 (2015), A1614-A1635.

[10] V.J. Ervin and J.P. Roop, Variational solution of fractional advection dispersion equations on bounded domains in $\mathbb{R}^{d}$, Numer. Methods. Partial Differential Eq 23 (2007), 256-281.

[11] J. Ferziger and M. Peric, Numerical methods in fluid dynamics. SpringerVerlag, Berlin, 1996.

[12] R.M. Gray, Toeplitz and Circulant Matrices: A Review, Foundations and Trends in Communications and Information Theory, 2(3) (2006), 155-239.

[13] D. Kolomenskiy, R. Nguyen van yen and K. Schneider, Analysis and discretization of the volume penalized Laplace operator with Neumann boundary conditions, Appl. Numer. Math., 95 (2015), 238-249.

[14] D. Kolomenskiy and K. Schneider, A Fourier spectral method for the NavierStokes equations with volume penalization for moving solid obstacles, J. Compt. Phy, 228(2009), 5687-5709.

[15] C. Li and H. Ding, Higher order finite difference method for the reaction and anomalous-diffusion equation, Appl. Math. Modell. 38 (2014), 38023821.

[16] F. Liu, V. Anh, and I. Turner, Numerical solution of the space fractional Fokker-Planck equation, J. Comput. Appl. Math. 166 (2004), 209-219. 
[17] V.E. Lynch, B.A. Carreras, D. del-Castillo-Negrete, K.M. FerreiraMejias, and H.R. Hicks, Numerical methods for the solution of partial differential equations of fractional order, J. Comput. Phys. 192 (2003) 406421.

[18] M.M. Meerschaert, D.A. Benson, and B. Baeumer, Multidimensional advection and fractional dispersion. Phys. Rev. E, 59 (1999), 5026-5028.

[19] M.M. Meerschaert, H.P. Scheffler, and C. Tadjeran, Finite difference methods for two-dimensional fractional dispersion equation, J. Comput. Phys., 211 (2006), 249-261.

[20] M.M. Meerschaert and C. Tadjeran, Finite difference approximations for fractional advection-dispersion flow equations, J. Comput. Appl. Math., 172 (2004) 65-77.

[21] R. Metzler and J. Klafter, The restaurant at the end of the random walk: recent developments in the description of anomalous transport by fractional dynamics, J. Phys. A Math. Gen., 37 (2004), R161-R208.

[22] C. Peskin, The immersed boundary method, Acta Numerica, 11 (2012), 479-517.

[23] I. Podlubny, Fractional Differential Equations, Academic Press, 1999.

[24] J.P. Roop, Computational aspects of FEM approximation of fractional advection dispersion equations on bounded domains in $\mathbb{R}^{2}$, J. Comput. Appl. Math., 193 (2006), 243-268.

[25] Y. Saad, Iterative methods for sparse linear systems, 2nd ed., SIAM, Rhode Island, 2003.

[26] Samko, S., A. Kilbas, and O. Marichev, Fractional Integrals and Derivatives: Theory and Applications, Gordon and Breach, London, 1993.

[27] C. Tadjeran and M.M. Meerschaert, A second-order accurate numerical method for the two-dimensional fractional diffusion equation, J. Comput. Phys., 220 (2007) 813-823.

[28] H. Wang and T.S. Basu, A fast finite difference method for twodimensional space-fractional diffusion equations, SIAM J. Sci. Comput., 34 (2012), A2444-A2458. 
[29] H. Wang and N. Du, A superfast-preconditioned iterative method for steady-state space-fractional diffusion equations, J. Comput. Phys., 240 (2013), 49-57.

[30] H. Wang, K. Wang, and T. Sircar, A direct $O\left(N \log ^{2} N\right)$ finite difference method for fractional diffusion equations, J. Comput. Phys., 229 (2010), 8095-8104. 